\title{
CONTROLS INSENSITIZING THE NORM OF THE SOLUTION OF A SEMILINEAR HEAT EQUATION IN UNBOUNDED DOMAINS
}

\author{
LUZ DE TERESA
}

\begin{abstract}
We consider a semilinear heat equation in an unbounded domain $\Omega$ with partially known initial data. The insensitizing problem consists in finding a control function such that some functional of the state is locally insensitive to the perturbations of these initial data. For bounded domains Bodart and Fabre proved the existence of insensitizing controls of the norm of the observation of the solution in an open subset of the domain. In this paper we prove similar results when $\Omega$ is unbounded. We consider the problem in bounded domains of the form $\Omega_{r}=\Omega \cap B_{r}$, where $B_{r}$ denotes the ball centered in zero of radius $r$. We show that for $r$ large enough the control proposed by Bodart and Fabre for the problem in $\Omega_{r}$, provides an insensitizing control for our problem in $\Omega$.
\end{abstract}

\section{Statement of the Problem}

Let $\Omega \subset \mathbb{R}^{n}, n \geq 1$ be an open unbounded set of class $C^{2}$ uniformly with boundary $\partial \Omega$ (see section 2.1 for a precise definition). Let $T>0, \omega$ and $\Theta$ be two open bounded subsets of $\Omega$. We consider the following parabolic semilinear system:

$$
\left\{\begin{array}{l}
z_{t}-\Delta z+f(z)=\xi+h \chi_{\omega} \text { in } Q=\Omega \times(0, T) \\
z=0 \text { on } \Sigma=\partial \Omega \times(0, T) \\
z(x, 0)=y^{0}(x)+\tau_{0} z^{0} \text { in } \Omega
\end{array}\right.
$$

where $f$ is a globally Lipschitz $C^{2}$ function defined on $\mathbb{R}$, with $f(0)=0$ and with bounded second derivative.

The state equation (1.1) has incomplete data in the following sense:

- $\xi$ and $y^{0}$ are given respectively in $L^{2}(Q)$ and $L^{2}(\Omega)$;

- $z^{0} \in L^{2}(\Omega)$ is unknown and $\left\|z^{0}\right\|_{L^{2}(\Omega)}=1$;

- $\tau_{0} \in \mathbb{R}$ is unknown and small enough;

- $h=h(x, t)$ is a control term to be determined in $L^{2}(\omega \times(0, T))$ and $\chi_{\omega}$ is the characteristic function of the set $\omega$ where the control is supported.

J. L. Lions [8] has introduced the notion of insensitizing control and later Bodart-Fabre [2] generalize it in the following way: Let $\Phi$ be a differentiable functional defined on the set of solutions of (1.1) and let $\varepsilon>0$; we say that

IMATE, UNAM; Circuito Exterior, C.U. 04510, D.F. México.

Email: deteresa@matem.unam.mx .

Received by the journal August 9, 1996. Revised December 12, 1996. Accepted for publication February 26, 1997.

(c) Société de Mathématiques Appliqués et Industrielles. Typeset by LATEX. 
the control $h, \varepsilon$-insensitizes $\Phi(z)$ if

$$
\left|\frac{\partial \Phi\left(z\left(x, t ; h, \tau_{0}\right)\right)}{\partial \tau_{0}}\right|_{\tau_{0}=0} \mid \leq \varepsilon
$$

This definition means that the functional $\Phi$ is locally " $\varepsilon$-insensitive" to the perturbation $\tau_{0} z^{0}$. There are of course many possible choices of $\Phi$ but insensitivity condition (1.2) is not of use unless it may be reformulated as a more explicit control problem. This is why it seems reasonable for $\Phi$ to be the square of the $L^{2}$ norm of the state in some observation subset $\Theta \subset \Omega$. When

$$
\Phi(z)=\frac{1}{2} \int_{0}^{T} \int_{\Theta} z^{2}(x, t) d x d t
$$

it can be proved (see [2] or Appendix A) that the condition of $\varepsilon$-insensitivity (1.2) is equivalent to an approximate control problem. This equivalence is given in the following proposition. Note that the original notion of insensitizing control proposed by Lions [8] (which corresponds to $\varepsilon=0$ in (1.2)) can be reformulated into a exact control problem, while the one given by Bodart and Fabre leads to an approximate control problem.

Proposition 1.1. Let $y$ and $q$ be the solutions of the following cascade system:

$$
\begin{aligned}
& \left\{\begin{array}{l}
y_{t}-\Delta y+f(y)=\xi+h \chi_{\omega} \text { in } Q \\
y=0 \text { on } \Sigma \\
y(., 0)=y^{0} \text { in } \Omega
\end{array}\right. \\
& \left\{\begin{array}{l}
-q_{t}-\Delta q+f^{\prime}(y) q=y \chi_{\Theta} \text { in } Q \\
q=0 \text { on } \Sigma \\
q(., T)=0 \text { in } \Omega
\end{array}\right.
\end{aligned}
$$

where $\chi_{\Theta}$ is the characteristic function of the observation subset $\Theta$.

Then the condition (1.2) of $\varepsilon$-insensitivity is equivalent to

$$
\|q(., 0)\|_{L^{2}(\Omega)} \leq \varepsilon .
$$

REMARK 1.2. Observe that equation (1.5) is solved backward in time. So in (1.6) we are asking for a control $h$ such that the corresponding solution of (1.5) enters the ball in $L^{2}(\Omega)$ of radius $\varepsilon$ centered in zero after a time interval of length $T$. This is precisely an approximate control problem. However, the control $h$ acts indirectly in the equation of $q$ through the variable $y$. This makes this approximate controllability problem technically more difficult. For classical approximate controllability in bounded domains we refer to $[6]$. The case of unbounded domains has been studied in [5].

When $\Omega$ is a bounded set, Bodart and Fabre [2] proved the following result:

Theorem 1.3. Assume that $\Omega$ is bounded. If $\omega \cap \Theta \neq \emptyset$ and the function $f$ is of class $C^{1}$ and globally Lipschitz, then for every $\varepsilon>0$ there exists $h \in L^{2}(\omega \times(0, T))$ E-insensitizing the functional (1.3).

Esaim: Cocv, June 1997, Vol. 2, Pr. 125-149 
In order to prove Theorem 1.3 Bodart and Fabre first show the approximate controllability of the double linear system with potentials:

$$
\begin{aligned}
& \left\{\begin{array}{l}
\zeta_{t}-\Delta \zeta+a(x, t) \zeta=h \chi_{\omega} \text { in } Q \\
\zeta=0 \text { on } \Sigma \\
\zeta(., 0)=0 \text { in } \Omega
\end{array}\right. \\
& \left\{\begin{array}{l}
-\eta_{t}-\Delta \eta+b(x, t) \eta=\zeta \chi_{\odot} \text { in } Q \\
\eta=0 \text { on } \Sigma \\
\eta(., T)=0 \text { in } \Omega
\end{array}\right.
\end{aligned}
$$

with $a(x, t), b(x, t) \in L^{\infty}(Q)$. Then, by a fixed point method they obtain the $\varepsilon$-insensitizing condition for the cascade system (1.4)-(1.5). This fixed point technique, based on Schauder's Theorem, uses the boundedness of $\Omega$ and the compactness of Sobolev's embeddings.

In this paper we prove the following results for unbounded sets $\Omega$.

THEOREM 1.4. Let $\Omega \subset \mathbb{R}^{n}$ be an open and unbounded set with boundary of class $C^{2}$ uniformly. Assume that $\omega \cap \Theta \neq \emptyset$, the function $f$ is of class $C^{2}$, globally Lipschitz and with bounded second derivative, $f(0)=0$. Suppose that the data $\xi \in L^{2}(Q)$ and $y^{0} \in L^{2}(\Omega)$ have compact support, then for every $\varepsilon>0$, there exists a control $h \in L^{2}(\omega \times(0, T))$, $\varepsilon$-insensitizing the functional (1.3).

In this theorem we assume, in particular, the data $\xi$ and $y^{0}$ to have compact support. This condition can be eliminated in space dimensions $1 \leq n \leq 6$.

TheOREM 1.5. Suppose that $1 \leq n \leq 6$. Assume that $\Omega, \omega, \Theta$ and $f$ satisfy the assumptions of Theorem 1.4. Then for every $\varepsilon>0, \xi \in L^{2}(Q)$ and $y^{0} \in L^{2}(\Omega)$ there exists a control $h \in L^{2}(\omega \times(0, T))$, E-insensitizing the functional (1.3).

Furthermore, if $n \geq 7$, the same holds for data $\xi \in L^{1}\left(0, T ; L^{2}(\Omega) \cap\right.$ $\left.L^{n / 2}(\Omega)\right)$ and $y^{0} \in L^{2}(\Omega) \cap L^{n / 2}(\Omega)$.

To prove Theorem 1.4 we use an approximation technique introduced in [5]: For data $\xi$ and $y^{0}$ with compact support we consider the problem in bounded sets of the form $\Omega_{r}=B_{r} \cap \Omega$, where $B_{r}$ denotes the ball centered at 0 and radius $r$. We show that the controls $h_{r}$ proposed in [2] are uniformly bounded in $L^{2}(\omega \times(0, T))$. This fact with some a priori estimates allow to prove that for $r$ large enough the control in the restricted domain provides an $\varepsilon$-insensitizing control for the functional (1.3) in the whole domain $\Omega$. Observe that the conditions on $f$ are more restrictive than those of Theorem 1.3 (one more bounded derivative is required). Since $\Omega$ is not bounded, we need to ask $f(0)=0$ in order to ensure that the solutions belong to $L^{2}(Q)$. As we will see the restrictions over the derivatives appear in a natural way when we estimate the norm

$$
\left\|q(., 0)-q_{r}(., 0)\right\|_{L^{2}\left(\Omega_{r}\right)}
$$

where $y, q, y_{r}$ and $q_{r}$ are the solutions of

$$
\left\{\begin{array}{l}
y_{t}-\Delta y+f(y)=\xi+h_{r} \chi_{\omega} \text { in } Q \\
y=0 \text { on } \Sigma \\
y(., 0)=y^{0} \text { in } \Omega
\end{array}\right.
$$




$$
\begin{aligned}
& \left\{\begin{array}{l}
-q_{t}-\Delta q+f^{\prime}(y) q=y \chi_{\Theta} \text { in } Q \\
q=0 \text { on } \Sigma \\
q(., T)=0 \text { in } \Omega
\end{array}\right. \\
& \left\{\begin{array}{l}
y_{r, t}-\Delta y_{r}+f\left(y_{r}\right)=\xi+h_{r} \chi_{\omega} \text { in } Q_{r} \\
y_{r}=0 \text { on } \Sigma_{r} \\
y_{r}(., 0)=y^{0} \text { in } \Omega_{r}
\end{array}\right. \\
& \left\{\begin{array}{l}
-q_{r, t}-\Delta q_{r}+f^{\prime}\left(y_{r}\right) q_{r}=y_{r} \chi_{\Theta} \text { in } Q_{r} \\
q_{r}=0 \text { on } \Sigma_{r} \\
q_{r}(., T)=0 \text { in } \Omega_{r}
\end{array}\right.
\end{aligned}
$$

respectively, and $Q_{r}=\Omega_{r} \times(0, T), \Sigma_{r}=\partial \Omega_{r} \times(0, T)$.

The paper is organized in the following way: In section 2 we study the existence and properties of the minima of some functional arising in the insensitizing control of linear system. In particular, section 2.2 is devoted to prove a uniform bound of these minima. In section 3 we prove the convergence of the solutions of system (1.12)-(1.13) in the restricted domain $Q_{r}$ to the solution in the global domain when the data $\xi$ and $y^{0}$ have compact support. In section 4 we prove Theorem 1.4. The proof of Theorem 1.5 is given by density arguments in section 5 . For the sake of clarity of the exposition some of the most technical proofs are included in section 6 at the end of the paper. In the Appendix we give an sketch of the proof of Proposition 1.1 .

We are going to consider the following hypotheses throughout the paper excluding section 5 in which [H5] is omitted:

(H1) $f \in C^{2}(\mathbb{R})$, globally Lipschitz, $f(0)=0,\left|f^{\prime \prime}\right|_{L^{\infty}}=M<\infty,\left|f^{\prime}\right|_{L^{\infty}}=$ $L<\infty$.

(H2) $\Omega \subset \mathbb{R}^{n}$ is an unbounded open set of class $C^{2}$ uniformly.

(H3) $\Theta, \omega \subset \Omega$, are bounded open sets such that $\Theta \cap \omega \neq \emptyset$.

(H4) $h \chi_{\omega} \in L^{2}\left(\mathbb{R}^{n} \times(0, T)\right)$.

(H5) $\xi \in L^{2}(Q)$ and $y^{0} \in L^{2}\left(\mathbb{R}^{n}\right)$ with compact support. Moreover, we fix $\rho>0$ such that supp $y^{0} \subset B_{\rho}, \operatorname{supp} \xi \subset\left(B_{\rho} \times(0, T)\right), \omega, \Theta \subset B_{\rho}$.

Observe that the regularity of the domain $\Omega$ is not essential for the results of this paper since we are asking homogeneous Dirichlet boundary conditions. Everything can be done in a connected, open and unbounded domain $\Omega$. Nevertheless, we prefer to work in an unbounded domain of class $C^{2}$ uniformly in order to use the regularity that the elliptic theory provides us and to avoid further technical developments. This is used, in particular, during the proof of Theorem 1.5 .

\section{Study of A FUNCTIONAL ARISING IN THE CONTROLLABILITY OF THE LINEARIZED SYSTEM}

\subsection{Preliminaries}

For the sake of completeness we recall, first of all, the definition of domain of class $C^{s}$ uniformly. We say that a domain $\Omega$ (bounded or not) is uniformly regular of class $C^{s}(s \geq 1$ ) (see [3] or [13]), if there exists an integer $r>0$ Esaim: COCV, JUNe 1997, Vol. 2, Pf. 125-149 
and sequences $\left\{N_{j}\right\}$ of open subsets of $\mathbb{R}^{n}$ and homeomorphisms $\left\{\psi_{j}\right\}$ of $N_{j}$ on the unit ball in $\mathbb{R}^{n}$ such that:

i) Any $(r+1)$ distinct sets $N_{j}$ have empty intersection;

ii) $\psi_{j}\left(N_{j} \cap \Omega\right)=\left\{x:|x|<1, x_{n}>0\right\}, \psi_{j}\left(N_{j} \cap \partial \Omega\right)=\left\{x:|x|<1, x_{n}=0\right\}$;

iii) If $N_{j}^{\prime}=\psi_{j}^{-1}(|x|<1 / 2), \cup_{j} N_{j}^{\prime}$ contains the $(1 / r)$-neighborhood of $\partial \Omega$;

iv) For $y \in N_{j}, x \in \psi_{j}\left(N_{j}\right)$ we have $\left|\left(D^{\alpha} \psi_{j}\right)(y)\right| \leq r,\left|\left(D^{\alpha} \psi_{j}^{-1}\right)(x)\right| \leq r$, for all $|\alpha| \leq s$.

For $0<t_{1}<t_{2}$ we denote by $X^{2}\left(t_{1}, t_{2} ; \Omega\right)$ the following Banach space:

$$
X^{2}\left(t_{1}, t_{2} ; \Omega\right)=L^{2}\left(t_{1}, t_{2} ; H_{0}^{1}(\Omega)\right) \cap H^{1}\left(t_{1}, t_{2} ; L^{2}(\Omega)\right)
$$

endowed with the natural norm

$$
\|\|_{X^{2}\left(t_{1}, t_{2} ; \Omega\right)}=\left(\|\|_{L^{2}\left(t_{1}, t_{2} ; H_{0}^{1}(\Omega)\right)}^{2}+\|\|_{H^{1}\left(t_{1}, t_{2} ; L^{2}(\Omega)\right)}^{2}\right)^{1 / 2} .
$$

Let $b(x, t) \in L^{\infty}(Q)$ and $0<t_{1}<t_{2}$. We recall (see [7], Theorem 9.1, page 341 ) the existence of constants $C>0$ (depending on $b, \Omega$ and $T$ ) and $C_{t_{1}, t_{2}}>0$ (depending of $b, \Omega, t_{1}$ and $t_{2}$ ) such that, for every $k \in L^{2}(Q)$ and $w^{0} \in L^{2}(\Omega)$, the solution $w$ of

$$
\left\{\begin{array}{l}
w_{t}-\Delta w+b(x, t) w=k \text { in } Q \\
w(x, t)=0 \text { on } \Sigma \\
w(x, 0)=w^{0}(x) \text { in } \Omega
\end{array}\right.
$$

satisfies

$$
\left\{\begin{array}{l}
\|w\|_{L^{\infty}\left(0, T ; L^{2}(\Omega)\right)} \leq C\left(\left\|w^{0}\right\|_{L^{2}(\Omega)}+\|k\|_{L^{2}(Q)}\right) \\
\|w\|_{X^{2}\left(t_{1}, t_{2} ; \Omega\right)} \leq C_{t_{1}, t_{2}}\left(\left\|w^{0}\right\|_{L^{2}(\Omega)}+\|k\|_{L^{2}(Q)}\right)
\end{array} .\right.
$$

Let us define now for $a_{r}(x, t), b_{r}(x, t) \in L^{\infty}\left(Q_{r}\right), \eta^{d} \in L^{2}\left(\Omega_{r}\right)$ and $\varphi_{r}^{0} \in$ $L^{2}\left(\Omega_{r}\right)$

$$
J_{r}\left(\varphi_{r}^{0} ; a_{r}, b_{r}, \eta^{d}\right)=\frac{1}{2} \int_{\omega \times(0, T)}\left|\psi_{r}\right|^{2} d x d t+\varepsilon\left\|\varphi_{r}^{0}\right\|_{L^{2}\left(\Omega_{r}\right)}-\int_{\Omega_{r}} \eta^{d} \varphi_{r}^{0} d x
$$

where $\varphi_{r}$ and $\psi_{r}$ are solutions of

$$
\begin{gathered}
\left\{\begin{array}{l}
\varphi_{r, t}-\Delta \varphi_{r}+b_{r}(x, t) \varphi_{r}=0 \text { in } Q_{r} \\
\varphi_{r}=0 \text { on } \Sigma_{r} \\
\varphi_{r}(., 0)=\varphi_{r}^{0} \text { in } \Omega_{r}
\end{array}\right. \\
\left\{\begin{array}{l}
-\psi_{r, t}-\Delta \psi_{r}+a_{r}(x, t) \psi_{r}=\varphi_{r} \chi_{\Theta} \text { in } Q_{r} \\
\psi_{r}=0 \text { on } \Sigma_{r} \\
\psi_{r}(., T)=0 \text { in } \Omega_{r} .
\end{array}\right.
\end{gathered}
$$

For each bounded subdomain $\Omega_{r}=\Omega \cap B_{r}$, that is for each $r>0$ fixed, Bodart and Fabre proved that $J_{r}\left(\cdot ; a_{r}, b_{r}, \eta^{d}\right)$ reaches its minimum at a unique point $\hat{\varphi}_{r}^{0} \in L^{2}\left(\Omega_{r}\right)$. Moreover, let $\left(\hat{\varphi}_{r}, \hat{\psi}_{r}\right)$ denote the solutions to $(2.2)-(2.3)$ corresponding to data $\hat{\varphi}_{r}^{0}$. If $\eta_{r}$ is the solution of (1.8) (in $Q_{r}, a=a_{r}, b=b_{r}$ ) corresponding to $h=\hat{\psi}_{r}$, then

$$
\left\|\eta_{r}(0)-\eta^{d}\right\|_{L^{2}\left(\Omega_{r}\right)} \leq \varepsilon
$$

That is $h=\hat{\psi}_{r}$ is an approximate control for the cascade linear problem in the truncated domains. 
For $\varphi^{0} \in L^{2}\left(\Omega_{r}\right)$ [resp. $\left.\varphi \in L^{2}\left(Q_{r}\right)\right]$ we denote by $\widetilde{\varphi^{0}}[$ resp. $\tilde{\varphi}$ ] the extension by zero of $\varphi^{0}$ [resp. $\varphi$ ] to $\Omega$ [resp. to $\left.Q\right]$, i.e.

$$
\widetilde{\varphi^{0}}=\left\{\begin{array}{ll}
\varphi^{0} & \text { in } \Omega_{r} \\
0 & \text { in } \Omega \backslash \Omega_{r}
\end{array}, \quad \widetilde{\varphi}= \begin{cases}\varphi & \text { in } Q_{r} \\
0 & \text { in } Q \backslash Q_{r} .\end{cases}\right.
$$

\subsection{UNIFORM BOUND ON THE MINIMIZERS}

The following result provides a uniform bound of the controls proposed in the previous section for the linear system in the truncated domains.

Proposition 2.1. Let $\left\{\tilde{a_{r}}\right\}_{r},\left\{\tilde{b_{r}}\right\}_{r}$ be bounded sequences in $L^{\infty}(Q)$. Suppose that $\left\{\tilde{\eta}_{r}^{d}\right\}_{r} \subset L^{2}(\Omega)$ converges strongly in $L^{2}(\Omega)$ to $\eta^{d}$ when $r \rightarrow \infty$. Then, there exists $C>0$ independent of $r$, such that

$$
\left\|\hat{\varphi}_{r}^{0}\right\|_{L^{2}\left(\Omega_{r}\right)} \leq C \text { for every } r>0
$$

and

$$
\left\|\hat{\psi}_{r}\right\|_{L^{2}\left(Q_{r}\right)} \leq C \text { for every } r>0
$$

where $\hat{\varphi}_{r}^{0}$ is the minimizer of $J_{r}\left(. ; a_{r}, b_{r}, \eta_{r}^{d}\right)$ and $\hat{\psi}_{r}$ is the corresponding solution to (2.3).

The proof of Proposition 2.1 needs the following two results. The first one is a unique continuation property, a consequence of a result due to Saut and Scheurer. For the proof see [11], Theorem 1.1. The second one is a consequence of a classical compactness result (see [12], Theorem 5). However since $\Omega$ is unbounded, its proof is technical and computations are long. To make the paper easier to read we give a detailed proof of Proposition 2.3 in section 6 at the end of the paper.

THEOREM 2.2. Let $\Omega \subset \mathbb{R}^{n}$ be an arbitrary open and connected subset. Let $Q=\Omega \times(0, T)$ and $a(x, t) \in L^{\infty}(Q)$. Let $\vartheta$ be an open and nonempty subset of $\Omega$ and $\varphi \in L_{l o c}^{2}\left(0, T ; H_{l o c}^{2}(\Omega)\right)$, such that

$$
\left\{\begin{array}{l}
-\varphi_{t}-\Delta \varphi+a(x, t) \varphi=0 \text { in } Q \\
\varphi=0 \text { in } \vartheta \times(0, T) .
\end{array}\right.
$$

Then,

$$
\varphi \equiv 0 \text { in } Q
$$

Proposition 2.3. Let $\left\{c_{r}\right\}_{r} \subset L^{\infty}\left(Q_{r}\right)$ be a sequence such that $\left\{\tilde{c}_{r}\right\}$ is bounded in $L^{\infty}(Q), \gamma_{r}^{0} \in L^{2}\left(\Omega_{r}\right)$ such that $\left\{\widetilde{\gamma_{r}^{0}}\right\}_{r}$ is bounded in $L^{2}(\Omega)$ and $g_{r} \in L^{\infty}\left(0, T ; L^{2}\left(\Omega_{r}\right)\right)$ with $\left\{\widetilde{g_{r}}\right\}_{r}$ bounded in $L^{\infty}\left(0, T ; L^{2}(\Omega)\right)$. Let $\gamma_{r}$ be the solution of the following system:

$$
\left\{\begin{array}{l}
\gamma_{r, t}-\Delta \gamma_{r}+c_{r}(x, t) \gamma_{r}=g_{r} \text { in } Q_{r} \\
\gamma_{r}=0 \text { on } \Sigma_{r} \\
\gamma_{r}(., 0)=\gamma_{r}^{0} \text { in } \Omega_{r}
\end{array}\right.
$$

Then, there exist $\gamma^{0} \in L^{2}(\Omega), g \in L^{2}(Q), \gamma \in L^{\infty}\left(0, T ; L^{2}(\Omega)\right) \cap C([0, T]$; $H_{l o c}^{-1}(\Omega)$ ), and a subsequence (still denoted by the subindex $r$ ) such that

$$
\begin{aligned}
& \widetilde{\gamma_{r}^{0}} \rightarrow \gamma^{0} \text { weakly in } L^{2}(\Omega) ; \\
& \widetilde{g_{r}} \rightarrow g \text { weakly in } L^{2}(Q) ;
\end{aligned}
$$




$$
\begin{gathered}
\tilde{\gamma_{r}} \rightarrow \gamma \text { weakly in } L^{2}(Q) ; \\
\widetilde{\gamma_{r}} \rightarrow \gamma \text { strongly in } L^{2}\left(\varepsilon, T ; L_{l o c}^{2}(\Omega)\right), \forall 0<\varepsilon<T ; \\
\widetilde{\gamma_{r}} \rightarrow \gamma \text { strongly in } C\left([0, T] ; H_{l o c}^{-1}(\Omega)\right) ; \\
\widetilde{\gamma_{r}}(t) \rightarrow \gamma(t) \text { strongly in } L_{l o c}^{2}(\Omega), \forall t \in(0, T]
\end{gathered}
$$

as $r \rightarrow \infty$. Moreover, $\gamma$ belongs to $L_{\text {loc }}^{2}\left(0, T ; H_{l o c}^{2}(\Omega)\right)$ and there exists $c=$ $c(x, t) \in L^{\infty}(Q)$ such that $\gamma=\gamma(x, t)$ satisfies:

$$
\gamma_{t}-\Delta \gamma+c(x, t) \gamma=g \text { in } Q
$$

in the sense of distributions.

Proof of Proposition 2.1. First of all we observe that (2.1) implies (2.5) once (2.4) is proved. For the proof of (2.4) we argue by contradiction.

It is clear that

$$
0=J_{r}\left(0 ; a_{r}, b_{r}, \eta_{r}^{d}\right) \geq J_{r}\left(\hat{\varphi}_{r}^{0} ; a_{r}, b_{r}, \eta_{r}^{d}\right) \text { for every } r .
$$

Suppose that there exists a subsequence $\left\{\hat{\varphi}_{r}^{0}\right\}_{r}$ such that

$$
\left\|\hat{\varphi}_{r}^{0}\right\|_{L^{2}\left(\Omega_{r}\right)} \rightarrow \infty \quad r \rightarrow \infty
$$

Let

$$
\mu_{r}^{0}=\frac{\hat{\varphi}_{r}^{0}}{\left\|\hat{\varphi}_{r}^{0}\right\|_{L^{2}\left(\Omega_{r}\right)}} .
$$

Then

$$
\begin{aligned}
\frac{J_{r}\left(\hat{\varphi}_{r}^{0} ; a_{r}, b_{r}, \eta_{r}^{d}\right)}{\left\|\hat{\varphi}_{r}^{0}\right\|_{L^{2}\left(\Omega_{r}\right)}} & =\left\|\hat{\varphi}_{r}^{0}\right\|_{L^{2}\left(\Omega_{r}\right)} \frac{1}{2} \int_{\omega \times(0, T)}\left|\beta_{r}(x, t)\right|^{2} d x d t \\
& +\varepsilon-\int_{\Omega_{r}} \eta_{r}^{d} \mu_{r}^{0} d x
\end{aligned}
$$

where $\mu_{r}$ and $\beta_{r}$ are solutions of system

$$
\begin{gathered}
\left\{\begin{array}{l}
\mu_{r, t}-\Delta \mu_{r}+b_{r}(x, t) \mu_{r}=0 \text { in } Q_{r} \\
\mu_{r}=0 \text { on } \Sigma_{r} \\
\mu_{r}(., 0)=\mu_{r}^{0} \text { in } \Omega_{r}
\end{array}\right. \\
\left\{\begin{array}{l}
-\beta_{r, t}-\Delta \beta_{r}+a_{r}(x, t) \beta_{r}=\mu_{r} \chi_{\Theta} \text { in } Q_{r} \\
\beta_{r}=0 \text { on } \Sigma_{r} \\
\beta_{r}(., T)=0 \text { in } \Omega_{r} .
\end{array}\right.
\end{gathered}
$$

It is clear that we can apply the results of Proposition 2.3 to the sequence $\mu_{r}$. Let us denote by $r$ the subsequence stated in Proposition 2.3 and let $\mu$ [resp. $\mu^{0}$ ] be the limit of $\mu_{r}$ [resp. $\mu_{r}^{0}$. Observe that if we put $t^{\prime}=T-t$ then $\beta_{r}\left(t^{\prime}\right)$ satisfies $(2.6)$ with $c_{r}\left(x, t^{\prime}\right)=a_{r}\left(x, t^{\prime}\right), g_{r}=\mu_{r} \chi_{\theta}$ and $\gamma_{r}^{0}=0$. Since $\left\|\mu_{r}^{0}\right\|_{L^{2}\left(\Omega_{r}\right)}=1$ by (2.1) we know that $\widetilde{\mu_{r}} \chi_{\theta}$ is uniformly bounded in $L^{\infty}\left(0, T ; L^{2}(\Omega)\right)$ and therefore $\beta_{r}$ verifies the hypotheses of Proposition 2.3. We can choose a subsequence $\beta_{r}$ (of that chosen for $\mu_{r}$ ) verifying the conclusions of Proposition 2.3. On the other hand, for $r$ large enough

$$
\int_{\omega \times(0, T)}\left|\widetilde{\beta}_{r}\right|^{2}=\int_{\omega \times(0, T)}\left|\beta_{r}\right|^{2}
$$


and therefore in view of (2.9) $\liminf _{r \rightarrow \infty} \int_{\omega \times(0, T)}\left|\beta_{r}\right|^{2} \geq \int_{\omega \times(0, T)}|\beta|^{2}$. Then by (2.14), (2.16) and since $\left\|\hat{\varphi}_{r}^{0}\right\|_{L^{2}\left(\Omega_{r}\right)} \rightarrow \infty$ necessarily

$$
\int_{\omega \times(0, T)}\left|\beta_{r}\right|^{2} \rightarrow 0 .
$$

Then $\beta \equiv 0$ in $\omega \times(0, T)$. But (2.13) asserts that $\beta$ verifies $-\beta_{t}-\Delta \beta+$ $a(x, t) \beta=\mu \chi_{\theta}$ for some $a \in L^{\infty}(Q)$. That is, $\mu=0$ in $\omega \cap \Theta \times(0, T)$.

Proposition 2.3 asserts that $\mu$ belongs to $L_{l o c}^{2}\left(0, T ; H_{l o c}^{2}(\Omega)\right)$ and since $\omega \cap \Theta \neq \emptyset$ we can apply the Unique Continuation Theorem 2.2 obtaining $\mu \equiv 0$ in $\Omega \times(\varepsilon, T)$. Moreover, by Proposition 2.3, we know that $\mu \in C\left([0, T] ; H_{l o c}^{-1}(\Omega)\right)$. Therefore

$$
\mu(0)=\mu^{0}=0 \text { in } \Omega .
$$

On the other hand,

$$
J_{r}\left(\hat{\varphi}_{r}^{0} ; a_{r}, b_{r}, \eta_{r}^{d}\right) \geq\left\|\hat{\varphi}_{r}^{0}\right\|_{L^{2}\left(\Omega_{r}\right)}\left(\varepsilon-\int_{\Omega} \widetilde{\eta_{r}^{d}} \widetilde{\mu_{r}^{0}} d x\right)
$$

and then $J_{r}\left(\hat{\varphi}_{r}^{0} ; a_{r}, b_{r}, \eta_{r}^{d}\right) \rightarrow \infty$, which contradicts (2.14). Therefore (2.4) is proved.

\section{Convergence of THE SOlutions DeFined in THE}

\section{APPROXIMATE DOMAINS}

The main purpose of this section is to prove the following result.

Proposition 3.1. Assume that (H1)-(H5) hold. Let $\left\{h_{r}\right\}_{r} \subset L^{2}(\omega \times(0, T))$ be a uniformly bounded sequence of controls. For each $r>0$ let $q_{r}$ be the solution to (1.13) corresponding to $h_{r}$ and $q=q\left(h_{r}\right)$ be the solution of (1.11) corresponding to the same control. Then $\left\|q(0)-q_{r}(0)\right\|_{L^{2}\left(\Omega_{r}\right)} \rightarrow 0$ as $r \rightarrow \infty$.

REMARK 3.2. In particular $\|q(0)\|_{L^{2}(\Omega)} \leq\left\|q(0)-q_{r}(0)\right\|_{L^{2}\left(\Omega_{r}\right)}+$ $\|q(0)\|_{L^{2}\left(\Omega \backslash \Omega_{r}\right)}+\left\|q_{r}(0)\right\|_{L^{2}\left(\Omega_{r}\right)}$. That is, given $\varepsilon>0$, if we can find controls $h_{r}$ verifying the hypotheses of Proposition 3.1 and such that $\left\|q_{r}(0)\right\|_{L^{2}\left(\Omega_{r}\right)} \leq \varepsilon / 2$ then for $r$ large enough $q=q\left(h_{r}\right)$ verifies $\|q(0)\|_{L^{2}(\Omega)} \leq \varepsilon$. That is, $h_{r}$ will be an $\varepsilon$-insensitizing control for the problem in the unbounded domain and in that case the main result for data with compact support (Theorem 1.4) would be proved.

In order to prove Proposition 3.1 we need some a priori estimates stated in the following lemmas.

Lemma 3.3. Assume that (H1)-(H5) hold. Let $y, q$ be the solutions of the cascade system:

$$
\begin{aligned}
& \left\{\begin{array}{l}
y_{t}-\Delta y+f(y)=\xi+h \chi_{\omega} \text { in } Q \\
y=0 \text { on } \Sigma \\
y(., 0)=y^{0} \text { in } \Omega
\end{array}\right. \\
& \left\{\begin{array}{l}
-q_{t}-\Delta q+f^{\prime}(y) q=y \chi_{\Theta} \text { in } Q \\
q=0 \text { on } \Sigma \\
q(., T)=0 \text { in } \Omega .
\end{array}\right.
\end{aligned}
$$

Then, there exists $C>0$ independent of $r$ such that for every $r>\rho$, Esaim: Cocv, June 1997, Vol. 2, Pr. 125-149 

i) $\|y\|_{L^{\infty}\left(\Gamma_{r}\right)} \leq \frac{C}{(r-\rho)^{n}}\left(\left\|y^{0}\right\|_{L^{1}(\Omega)}+\|\xi\|_{L^{1}(Q)}+\|h\|_{L^{1}(\omega \times(0, T))}\right)$
ii) $\|q\|_{L^{\infty}\left(\Gamma_{r}\right)} \leq \frac{C}{(r-\rho)^{n}}\left(\left\|y^{0}\right\|_{L^{2}(\Omega)}+\|\xi\|_{L^{2}(Q)}+\|h\|_{L^{2}(\omega \times(0, T))}\right)$.

Moreover, since supp $y^{0} \subset B_{\rho}$, supp $\xi \subset B_{\rho} \times(0, T), \omega \subset B_{\rho}$ we have that

iii) $\|y\|_{L^{\infty}\left(\Gamma_{r}\right)} \leq \frac{C}{(r-\rho)^{n}}\left(\left\|y^{0}\right\|_{L^{2}(\Omega)}+\|\xi\|_{L^{2}(Q)}+\|h\|_{L^{2}(\omega \times(0, T))}\right)$.

Lemma 3.4. Assume that $g \in L^{\infty}\left(\Gamma_{r}\right)$, with $\Gamma_{r}=\partial B_{r} \times(0, T)$. Let $w$ satisfy:

$$
\left\{\begin{array}{l}
w_{t}-\Delta w=0 \text { in } B_{r} \times(0, T) \\
w=g \text { on } \Gamma_{r} \\
w(x, 0)=0 \text { in } B_{r} .
\end{array}\right.
$$

Then, there exists $C>0$ independent of $r$ such that

$$
\|w\|_{L^{2}\left(B_{r} \times(0, T)\right)} \leq C(T) r^{n / 2}\|g\|_{L^{\infty}\left(\Gamma_{r}\right)} \forall r>0
$$

and, in particular,

$$
\|w(T)\|_{L^{2}\left(B_{r}\right)} \leq C r^{n / 2}\|g\|_{L^{\infty}\left(\Gamma_{r}\right)} \forall r>0 .
$$

Let us assume that Lemma 3.3 and Lemma 3.4 hold in order to prove Proposition 3.1. The proof of these lemmas will be given at the end of this section.

Proof of Proposition 3.1. Let $\psi=y-y_{r}$ with $y$ solution of (1.10) and $y_{r}$ solution of (1.12) both corresponding to the control $h_{r}, \beta=q-q_{r}$ with $q$ solution of (1.11) and $q_{r}$ solution of (1.13). Then $\psi$ and $\beta$ are solutions of the following system:

$$
\begin{gathered}
\left\{\begin{array}{l}
\psi_{t}-\Delta \psi+f(y)-f\left(y_{r}\right)=0 \text { in } Q_{r} \\
\psi=y \text { on } \Sigma_{r} \\
\psi(., 0)=0 \text { in } \Omega_{r},
\end{array}\right. \\
\left\{\begin{array}{l}
-\beta_{t}-\Delta \beta+f^{\prime}(y) q-f^{\prime}\left(y_{r}\right) q_{r}=\psi \chi_{\Theta} \text { in } Q_{r} \\
\beta=q \text { on } \Sigma_{r} \\
\beta(., T)=0 \text { in } \Omega_{r} .
\end{array}\right.
\end{gathered}
$$

We write $\beta=v+\gamma$ where $\gamma$ and $v$ verify, respectively,

$$
\begin{aligned}
& \left\{\begin{array}{l}
-\gamma_{t}-\Delta \gamma+f^{\prime}(y) q-f^{\prime}\left(y_{r}\right) q_{r}=\psi \chi_{\Theta} \text { in } Q_{r} \\
\gamma=0 \text { on } \Sigma_{r} \\
\gamma(., T)=0 \text { in } \Omega_{r} .
\end{array}\right. \\
& \left\{\begin{array}{l}
-v_{t}-\Delta v=0 \text { in } Q_{r} \\
v=q \text { on } \Sigma_{r} \\
v(., T)=0 \text { in } \Omega_{r} .
\end{array}\right.
\end{aligned}
$$

In order to estimate the norm of $\beta(0)$ in $L^{2}\left(\Omega_{r}\right)$ we are going to estimate the norms of $v(0)$ and $\gamma(0)$ in $L^{2}\left(\Omega_{r}\right)$. Nevertheless, as we shall see, in order to estimate the norm of $\gamma(0)$ it is necessary to estimate the norm of $v$ in $L^{2}\left(Q_{r}\right)$. That is the first thing we are going to do.

Let $\hat{w}$ be the solution of

$$
\left\{\begin{array}{l}
-\hat{w}_{t}-\Delta \hat{w}=0 \text { in } B_{r} \times(0, T) \\
\hat{w}=\left\{\begin{array}{l}
|q| \text { on }\left(\partial \Omega_{r} \cap \partial B_{r}\right) \times(0, T)=\Sigma_{r}^{\prime} \\
0 \text { on } \Gamma_{r} \backslash \Sigma_{r}^{\prime}
\end{array}\right. \\
\hat{w}(., T)=0 \text { in } B_{r},
\end{array}\right.
$$


where we recall that $\Gamma_{r}$ denotes $\partial B_{r} \times(0, T)$.

Since $q=0$ on $\Sigma_{r} \backslash \Sigma_{r}^{\prime}$, by the maximum principle,

$$
\|v\|_{L^{2}\left(Q_{r}\right)} \leq\|\hat{w}\|_{L^{2}\left(B_{r} \times(0, T)\right)}
$$

but in view of Lemma 3.5 (noticing that $\hat{w}\left(t^{\prime}\right)$ satisfies $(3.3)$, with $t^{\prime}=T-t$ )

$$
\|\hat{w}\|_{L^{2}\left(B_{r} \times(0, T)\right)} \leq C r^{n / 2}\|q\|_{L^{\infty}\left(\Sigma_{r}\right)}
$$

and

That is,

$$
\|\hat{w}(0)\|_{L^{2}\left(B_{r}\right)} \leq C r^{n / 2}\|q\|_{L^{\infty}\left(\Sigma_{r}\right)} .
$$

$$
\|v\|_{L^{2}\left(Q_{r}\right)} \leq C r^{n / 2}\|q\|_{L^{\infty}\left(\Sigma_{r}\right)}
$$

and

$$
\|v(0)\|_{L^{2}\left(\Omega_{r}\right)} \leq C r^{n / 2}\|q\|_{L^{\infty}\left(\Sigma_{r}\right)}
$$

with $C>0$ a generic constant independent of $r$.

In order to estimate $\|\gamma(0)\|_{L^{2}\left(\Omega_{r}\right)}$ we multiply (3.6) by $\gamma$ and integrate by parts:

$-\frac{1}{2} \frac{d}{d t} \int_{\Omega_{r}}|\gamma|^{2} d x+\int_{\Omega_{r}}|\nabla \gamma|^{2} d x=\int_{\Omega_{r}}\left(f^{\prime}\left(y_{r}\right) q_{r}-f^{\prime}(y) q\right) \gamma d x+\int_{\Omega_{r}} \psi \gamma_{\Theta} d x$.

We observe that

$$
f^{\prime}(y) q-f^{\prime}\left(y_{r}\right) q_{r}=q\left(f^{\prime}(y)-f^{\prime}\left(y_{r}\right)\right)+f^{\prime}\left(y_{r}\right)\left(q-q_{r}\right)
$$

and then

$$
\begin{aligned}
& -\frac{1}{2} \frac{d}{d t} \int_{\Omega_{r}}|\gamma|^{2} d x+\int_{\Omega_{r}}|\nabla \gamma|^{2} d x \\
& \leq M \int_{\Omega_{r}}|q \psi \gamma| d x+L \int_{\Omega_{r}}|\gamma+v||\gamma| d x+\int_{\Omega_{r}} \psi \gamma \chi_{\Theta} d x
\end{aligned}
$$

with $M \geq\left\|f^{\prime \prime}\right\|_{L^{\infty}}, L=\left\|f^{\prime}\right\|_{L^{\infty}}$.

We conclude, by Schwartz's and Gronwall's inequalities, that

$$
\|\gamma(0)\|_{L^{2}\left(\Omega_{r}\right)} \leq C\left(\|\psi\|_{L^{2}\left(Q_{r}\right)}+\|q \psi\|_{L^{2}\left(Q_{r}\right)}+\|v\|_{L^{2}\left(Q_{r}\right)}\right),
$$

with the constant $C$ independent of $r$. In view of (3.7), (3.8) and (3.9) we obtain that

$$
\|\beta(0)\|_{L^{2}\left(\Omega_{r}\right)} \leq C\left(r^{n / 2}\|q\|_{L^{\infty}\left(\Sigma_{r}\right)}+\|\psi\|_{L^{2}\left(Q_{r}\right)}+\|q \psi\|_{L^{2}\left(Q_{r}\right)}\right) .
$$

We proceed now to estimate $\|q \psi\|_{L^{2}\left(Q_{r}\right)}$. In this aim we observe that since $y$ belongs to $L^{\infty}\left(\Sigma_{r}\right)$ by the maximum principle we see that $\psi \in L^{\infty}\left(Q_{r}\right)$ and $\|\psi\|_{L^{\infty}\left(Q_{r}\right)} \leq C\|y\|_{L^{\infty}\left(\Sigma_{r}\right)}$ where $C>0$ does not depend of $r$. That implies that

$$
\|q \psi\|_{L^{2}\left(Q_{r}\right)} \leq C\|q\|_{L^{2}\left(Q_{r}\right)}\|y\|_{L^{\infty}\left(\Sigma_{r}\right)} \leq C\|q\|_{L^{2}(Q)}\|y\|_{L^{\infty}\left(\Sigma_{r}\right)}
$$

Moreover, proceeding as in the proof of $(3.10)$, we can prove that there exists $C>0$, such that

$$
\|\psi\|_{L^{2}\left(Q_{r}\right)} \leq C r^{n / 2}\|y\|_{L^{\infty}\left(\Sigma_{r}\right)}
$$

On the other hand, multiplying (1.5) by $q$ and (1.4) by $y$ and integrating by parts, it is easy to see that

$$
\|q\|_{L^{2}(Q)} \leq C\left(\left\|y^{0}\right\|_{L^{2}(\Omega)}+\|\xi\|_{L^{2}(Q)}+\left\|h_{r} \chi_{\omega}\right\|_{L^{2}(Q)}\right)
$$


From (3.11), (3.12), (3.13) and (3.10) we obtain

$$
\begin{aligned}
\|\beta(0)\|_{L^{2}\left(\Omega_{r}\right)} \leq & C\left[r^{n / 2}\|y\|_{L^{\infty}\left(\Sigma_{r}\right)}+\right. \\
& \left(\left\|y^{0}\right\|_{L^{2}(\Omega)}+\|\xi\|_{L^{2}(Q)}+\left\|h_{r} \chi_{\omega}\right\|_{L^{2}(Q)}\right)\|y\|_{L^{\infty}\left(\Sigma_{r}\right)} \\
& \left.+r^{n / 2}\|q\|_{L^{\infty}\left(\Sigma_{r}\right)}\right] .
\end{aligned}
$$

In view of Lemma 3.3, we obtain, for every $r>\rho$, that

$$
\|\beta(0)\|_{L^{2}\left(\Omega_{r}\right)} \leq C\left[\frac{r^{n / 2}}{(r-\rho)^{n}}\left(1+\left\|y^{0}\right\|_{L^{2}(\Omega)}+\|\xi\|_{L^{2}(Q)}+\left\|h_{r} \chi_{\omega}\right\|_{L^{2}(Q)}\right)^{2}\right] .
$$

Since the sequence $h_{r}$ is uniformly bounded, we conclude the proof by noticing that the right hand side converges to zero as $r$ goes to $\infty$.

We proceed now to prove Lemma 3.3. In this aim we use the following estimate for the solutions of the cascade system in $\mathbb{R}^{n}$ that is going to be proved in section 6 at the end of the paper:

LEMMA 3.5. Consider $F$ a globally Lipschitz function of class $C^{1}$ with $F(0)=0$. Assume that $y^{0} \in L^{2}\left(\mathbb{R}^{n}\right)$ and $g \in L^{2}\left(\mathbb{R}^{n} \times(0, T)\right)$ have compact support. We fix $\rho>0$ such that supp $y^{0} \subset B_{\rho}$, supp $g \subset B_{\rho} \times(0, T)$. Let $X$ be the solution of the following system:

$$
\left\{\begin{array}{l}
X_{t}-\Delta X+F(X)=|g| \text { in } \mathbb{R}^{n} \times(0, T) \\
X(., 0)=\left|y^{0}\right| \text { in } \mathbb{R}^{n} .
\end{array}\right.
$$

Then, there exists a constant $C>0$ independent of $r$, such that for every $r>\rho$,

$$
|X|_{L^{\infty}\left(\Gamma_{r}\right)} \leq \frac{C}{(r-\rho)^{n}}\left(\left\|y^{0}\right\|_{L^{1}\left(\mathbb{R}^{n}\right)}+\|g\|_{L^{1}\left(\mathbb{R}^{n} \times(0, T)\right)}\right)
$$

where $\Gamma_{r}$ denotes $\partial B_{r} \times(0, T)$.

REMARK 3.6. Following the same procedure it is easy to see that if $\tilde{X}$ satisfies

$$
\left\{\begin{array}{l}
\tilde{X}_{t}-\Delta \tilde{X}+F(\tilde{X})=-|g| \text { in } \mathbb{R}^{n} \times(0, T) \\
\tilde{X}(., 0)=-\left|y^{0}\right| \text { in } \mathbb{R}^{n}
\end{array}\right.
$$

then, for every $r>\rho$ :

$$
\|\tilde{X}\|_{L^{\infty}\left(\Gamma_{r}\right)} \leq \frac{C}{(r-\rho)^{n}}\left(\left\|y^{0}\right\|_{L^{1}\left(\mathbb{R}^{n}\right)}+\|g\|_{L^{1}\left(\mathbb{R}^{n} \times(0, T)\right)}\right) .
$$

Proof of Lemma 3.3. Let $S=Y-y, R=Z-q$ where $Y$ and $Z$ solve

$$
\begin{gathered}
\left\{\begin{array}{l}
Y_{t}-\Delta Y+f(Y)=|\xi|+|h| \chi_{\omega} \text { in } \mathbb{R}^{n} \times(0, T) \\
Y(., 0)=\left|y^{0}\right| \text { in } \mathbb{R}^{n}
\end{array}\right. \\
\left\{\begin{array}{l}
-Z_{t}-\Delta Z-L Z=Y \chi_{\Theta} \text { in } \mathbb{R}^{n} \times(0, T) \\
Z(., T)=0 \text { in } \mathbb{R}^{n},
\end{array}\right.
\end{gathered}
$$

where $L=\left|f^{\prime}\right|_{\infty}$.

If we denote by $\xi^{-}$and $h^{-}$the negative parts of $\xi$ and $h$, respectively, then $S$ and $R$ satisfy

$$
\left\{\begin{array}{l}
S_{t}-\Delta S+f(Y)-f(y)=2 \xi^{-}+2 h^{-} \chi_{\omega} \text { in } Q \\
S=Y \text { on } \Sigma \\
S(., 0)=2 y^{0^{-}} \text {on } \Omega
\end{array}\right.
$$




$$
\left\{\begin{array}{l}
-R_{t}-\Delta R-L Z-f^{\prime}(y) q=S \chi_{\Theta} \text { in } Q \\
R=Z \text { on } \Sigma \\
R(., T)=0 \text { in } \Omega
\end{array}\right.
$$

We multiply (3.17) by $-S^{-}$and integrate by parts:

$$
-\int_{\Omega} S_{t} S^{-}+\int_{\Omega} \Delta S S^{-}-\int_{\Omega}(f(Y)-f(y)) S^{-}=-\int_{\Omega} 2\left(\xi^{-}+h^{-} \chi_{\omega}\right) S^{-} .
$$

Since $S=Y$ on $\Sigma, Y \geq 0$ and $f$ is globally Lipschitz

$$
\frac{1}{2} \frac{d}{d t} \int_{\Omega}\left(S^{-}\right)^{2}+\int_{\Omega}\left(\nabla S^{-}\right)^{2} \leq L \int_{\Omega}\left(S^{-}\right)^{2} .
$$

Then, by Gronwall's inequality and since $S(0)^{-}=0$, we obtain that

$$
\int_{\Omega}\left(S^{-}(t)\right)^{2} \leq e^{2 L t} \int_{\Omega}\left(S(0)^{-}\right)^{2}-e^{2 L t} \int_{0}^{t} \int_{\Omega} e^{-2 L \sigma}\left(\nabla S(\sigma)^{-}\right)^{2} \leq 0, \forall t \in(0, T)
$$

and therefore

$$
S(x, t) \geq 0, \forall t \in(0, T), \text { a.e } x \in \Omega .
$$

We multiply (3.18) by $-R^{-}$and integrate by parts:

$$
\int_{\Omega} R_{t} R^{-}+\int_{\Omega} \Delta R R^{-}+\int_{\Omega}\left(f^{\prime}(y) q+L Z\right) R^{-}=-\int_{\Omega} S R^{-} \chi_{\Theta} .
$$

Since $R=Z$ on $\Sigma, Z \geq 0$ and $S R^{-} \geq 0$ for almost every $x \in \Omega$, we obtain

$$
-\frac{1}{2} \frac{d}{d t} \int_{\Omega}\left(R^{-}\right)^{2}+\int_{\Omega}\left(\nabla R^{-}\right)^{2} \leq \int_{\Omega}\left(-f^{\prime}(y) q-L Z\right) R^{-} .
$$

Since $Z \geq 0,-f^{\prime}(y) q-L Z \leq L|q|-L Z \leq L|R|$, by Gronwall's inequality

$$
\int_{\Omega}\left(R^{-}(t)\right)^{2} \leq 0, \forall t \in(0, T)
$$

and therefore

$$
R(x, t) \geq 0, \forall t \in(0, T) \text {, a.e } x \in \Omega .
$$

That implies that

$$
y(x, t) \leq Y(x, t) ; q(x, t) \leq Z(x, t), \forall t \in(0, T), \text { a.e. } x \in \Omega .
$$

In the same way we can prove that

$$
\tilde{Y}(x, t) \leq y(x, t) ; \tilde{Z}(x, t) \leq q(x, t), \forall t \in(0, T), \text { a.e } x \in \Omega
$$

where $\tilde{Y}$ is the solution of (3.15) corresponding to data $-|\xi|,-\left|y^{0}\right|$ and control $-|h| \chi_{\omega}$, and $\tilde{Z}$ solves (3.16) when substituting $Y$ by $\tilde{Y}$.

We conclude the proof of i) by applying the results of Lemma 3.5 and Remark 3.6 to $Y$ and $\tilde{Y}$. In order to obtain ii) we observe that we can apply the results of Lemma 3.5 and Remark 3.6 to $Z$ since if we put $t^{\prime}=T-t$ then $Z\left(t^{\prime}\right)$ satisfies (3.14) with $y^{0}=0, g=Y_{\chi_{\omega}}$ and $F(Z)=-L Z$. On the other hand,

$$
\left\|Y \chi_{\Theta}\right\|_{L^{1}\left(\mathbb{R}^{n} \times(0, T)\right)} \leq T^{1 / 2}|\Theta|^{1 / 2}\|Y\|_{L^{2}\left(\mathbb{R}^{n} \times(0, T)\right)} .
$$

Then, applying (2.1) to (3.15) we conclude ii). We obtain iii) from i) by noticing that ( $\mathrm{H} 5$ ) allows to find a constant independent of $r$ such that the $L^{1}$ norms of the data and the control are upper bounded by that constant times their respective $L^{2}$ norms. 
Proof of Lemma 3.4. Consider first the case where $r=1$ and suppose that $w$ solves $(3.3)$ with $r=1$. Since $g \in L^{\infty}\left(\Gamma_{1}\right)$, by the maximum principle we know that

$$
w(t) \in L^{2}\left(B_{1}\right) \text { for every } t \in(0, T)
$$

We consider the following transposed problem: for $\eta^{0} \in L^{2}\left(B_{1}\right)$ and $t \in$ $(0, T)$, let $\eta$ be the solution of the equation

$$
\left\{\begin{array}{l}
-\eta_{t}-\Delta \eta=0 \text { in } B_{1} \times(0, t) \\
\eta=0 \text { on } \Gamma_{1} \\
\eta(x, t)=\eta^{0} \text { in } B_{1}
\end{array}\right.
$$

Since $\eta_{0} \in L^{2}\left(B_{1}\right)$ we have that $\|\eta(t)\|_{H^{s}\left(B_{1}\right)} \leq t^{-s / 2}\left\|\eta^{0}\right\|_{L^{2}\left(B_{1}\right)}$ for $0<s<2$ (For the proof see Lions-Magenes [9]). Now, if $3 / 2<s<2$, we have that $\frac{\partial \eta}{\partial \nu}(t)$ belongs to $L^{1}\left(\partial B_{1}\right)$ [see Triebel [14], p. 330], and

$$
\left\|\frac{\partial \eta}{d \nu}(t)\right\|_{L^{1}\left(\partial B_{1}\right)} \leq C t^{-s / 2}\left\|\eta^{0}\right\|_{L^{2}\left(B_{1}\right)}
$$

and then

$$
\left\|\frac{\partial \eta}{d \nu}\right\|_{L^{1}\left(\partial B_{1} \times(0, t)\right)} \leq C\left(B_{1}, s\right) t^{(1-s / 2)}\left\|\eta^{0}\right\|_{L^{2}\left(B_{1}\right)} \quad \forall t \in(0, T) .
$$

Multiplying (3.3) by $\eta$ we obtain:

$$
\int_{B_{1}} w(t) \eta^{0}=\int_{0}^{t} \int_{\partial B_{1}} \frac{\partial \eta}{\partial \nu} g
$$

and in view of $(3.20)$ we have

$$
\left|\int_{B_{1}} w(t) \eta^{0}\right| \leq\left\|\frac{\partial \eta}{\partial \nu}\right\|_{L^{1}\left(\Gamma_{1}\right)}\|g\|_{L^{\infty}\left(\Gamma_{1}\right)} \leq C\left(B_{1}\right) t^{\varepsilon / 2}\left\|\eta^{0}\right\|_{L^{2}\left(B_{1}\right)}\|g\|_{L^{\infty}\left(\Gamma_{1}\right)} .
$$

Therefore

$$
\|w(t)\|_{L^{2}\left(B_{1}\right)} \leq C\left(B_{1}\right) t^{\varepsilon / 2}\|g\|_{L^{\infty}\left(\Gamma_{1}\right)}
$$

where $\frac{1}{2}>\varepsilon=2-s, 2>s>3 / 2$ and $C\left(B_{1}\right)$ is independent of $t$.

Let now $w$ be the solution of $(3.3), \bar{w}(x, t)=w\left(r x, r^{2} t\right)$ and $\bar{g}(x, t)=$ $g\left(r x, r^{2} t\right)$. Then $\bar{w}$ satisfies

$$
\left\{\begin{array}{l}
\bar{w}_{t}-\Delta \bar{w}=0 \text { in } B_{1} \times\left(0, T / r^{2}\right) \\
\bar{w}=\bar{g} \text { on } \partial B_{1} \times\left(0, T / r^{2}\right)=\bar{\Gamma}_{1} \\
\bar{w}(x, 0)=0 \text { in } B_{1}
\end{array}\right.
$$

In view of (3.21)

$$
\left\|\bar{w}\left(\frac{t}{r^{2}}\right)\right\|_{L^{2}\left(B_{1}\right)} \leq C\left(\frac{t}{r^{2}}\right)^{\varepsilon / 2}\|\bar{g}\|_{L^{\infty}\left(\bar{\Gamma}_{1}\right)} \quad \forall t \in[0, T]
$$

and then

$$
\int_{B_{r}} w^{2}(x, t) d x \leq r^{n} C\left(\frac{t}{r^{2}}\right)^{\varepsilon}\|g\|_{L^{\infty}\left(\Gamma_{r}\right)}^{2}
$$

That is,

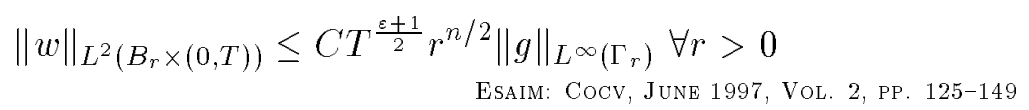


where $C$ is independent of $r$. In particular, we have obtained

$$
\|w(T)\|_{L^{2}\left(B_{r}\right)} \leq C r^{n / 2}\|g\|_{L^{\infty}\left(\Gamma_{r}\right)} \forall r>0 .
$$

\section{Study OF THE CONTROLS FOR THE RESTRICTED DOMAINS}

First of all we recall the controls proposed in [2]. We introduce

$$
F(s)=\left\{\begin{array}{r}
\frac{f(s)}{s} \text { if } s \neq 0 \\
f^{\prime}(0) \text { if } s=0 .
\end{array}\right.
$$

Since $f$ is of class $C^{2}$ and globally Lipschitz, $F \in L^{\infty}(\mathbb{R})$.

Let $\varepsilon>0, y^{0} \in L^{2}(\Omega), \xi \in L^{2}(Q)$ satisfying (H5). For $r>\rho$ fixed and given $z_{r} \in L^{2}\left(Q_{r}\right)$, we write $y_{r}=\zeta_{r}+\bar{\zeta}_{r}, q_{r}=\eta_{r}+\bar{\eta}_{r}$ with $\bar{\zeta}_{r}, \bar{\eta}_{r}$ solutions of

$$
\begin{gathered}
\left\{\begin{array}{l}
\bar{\zeta}_{r, t}-\Delta \bar{\zeta}_{r}+F\left(z_{r}\right) \bar{\zeta}_{r}=\xi \text { in } Q_{r} \\
\bar{\zeta}_{r}=0 \text { on } \Sigma_{r} \\
\bar{\zeta}_{r}(., 0)=y^{0} \text { in } \Omega_{r}
\end{array}\right. \\
\left\{\begin{array}{l}
-\bar{\eta}_{r, t}-\Delta \bar{\eta}_{r}+f^{\prime}\left(z_{r}\right) \bar{\eta}_{r}=\bar{\zeta}_{r} \chi_{\Theta} \text { in } Q_{r} \\
\bar{\eta}_{r}=0 \text { on } \Sigma_{r} \\
\bar{\eta}_{r}(., T)=0 \text { in } \Omega_{r} .
\end{array}\right.
\end{gathered}
$$

In [2] it is proved that there exists $\psi_{r} \in L^{2}\left(Q_{r}\right)$, such that if $\zeta_{r}, \eta_{r}$ are the solutions of

$$
\begin{aligned}
& \left\{\begin{array}{l}
\zeta_{r, t}-\Delta \zeta_{r}+F\left(z_{r}\right) \zeta_{r}=\psi_{r} \chi_{\omega} \text { in } Q_{r} \\
\zeta_{r}=0 \text { on } \Sigma_{r} \\
\zeta_{r}(., 0)=0 \text { in } \Omega_{r}
\end{array}\right. \\
& \left\{\begin{array}{l}
-\eta_{r, t}-\Delta \eta_{r}+f^{\prime}\left(z_{r}\right) \eta_{r}=\zeta_{r} \chi_{\Theta} \text { in } Q_{r} \\
\eta_{r}=0 \text { on } \Sigma_{r} \\
\eta_{r}(., T)=0 \text { in } \Omega_{r} .
\end{array}\right.
\end{aligned}
$$

Then $\left\|\eta_{r}(0)+\bar{\eta}_{r}(0)\right\|_{L^{2}\left(\Omega_{r}\right)} \leq \varepsilon$. That is,

$$
\left\|q_{r}(0)\right\|_{L^{2}\left(\Omega_{r}\right)} \leq \varepsilon .
$$

Moreover, $\varphi_{r}, \psi_{r}$ satisfy

$$
\begin{aligned}
& \left\{\begin{array}{l}
\varphi_{r, t}-\Delta \varphi_{r}+f^{\prime}\left(z_{r}\right) \varphi_{r}=0 \text { in } Q_{r} \\
\varphi_{r}=0 \text { on } \Sigma_{r} \\
\varphi_{r}(., 0)=\varphi_{r}^{0} \text { in } \Omega_{r}
\end{array}\right. \\
& \left\{\begin{array}{l}
-\psi_{r, t}-\Delta \psi_{r}+F\left(z_{r}\right) \psi_{r}=\varphi_{r} \chi_{\Theta} \text { in } Q_{r} \\
\psi_{r}=0 \text { on } \Sigma_{r} \\
\psi_{r}(., T)=0 \text { in } \Omega_{r} .
\end{array}\right.
\end{aligned}
$$

with $\varphi_{r}^{0} \in L^{2}\left(\Omega_{r}\right)$ the corresponding minimizer of $J_{r}\left(. ; F\left(z_{r}\right), f^{\prime}\left(z_{r}\right),-\bar{\eta}_{r}(0)\right)$.

Let us consider the nonlinear mapping:

$$
\Lambda_{r}: L^{2}\left(Q_{r}\right) \rightarrow L^{2}\left(Q_{r}\right),
$$

$$
\Lambda_{r}\left(z_{r}\right)=\left\{y_{r}\left(z_{r}, \psi_{r}\left(z_{r}\right)\right) ;\left\|q_{r}(0)\right\|_{L^{2}\left(\Omega_{r}\right)} \leq \varepsilon ; \varphi_{r}^{0} \text { minimizing } J_{r}\right\} .
$$


In [2] was proved that $\Lambda_{r}$ has a fixed point such that

$$
\left\{\begin{array}{l}
\varphi_{r, t}-\Delta \varphi_{r}+f^{\prime}\left(y_{r}\right) \varphi_{r}=0 \text { in } Q_{r} \\
\varphi_{r}=0 \text { on } \Sigma_{r} \\
\varphi_{r}(., 0)=\varphi_{r}^{0} \text { in } \Omega_{r} \\
-\psi_{r, t}-\Delta \psi_{r}+F\left(y_{r}\right) \psi_{r}=\varphi_{r} \chi_{\Theta} \text { in } Q_{r} \\
\psi_{r}=0 \text { on } \Sigma_{r} \\
\psi_{r}(., T)=0 \text { in } \Omega_{r} \\
y_{r, t}-\Delta y_{r}+f\left(y_{r}\right)=\xi+\psi_{r} \chi_{\omega} \text { in } Q_{r} \\
y_{r}=0 \text { on } \Sigma_{r} \\
y_{r}(., 0)=y^{0} \chi_{\Omega_{r}} \text { in } \Omega_{r} \\
-q_{r, t}-\Delta q_{r}+f^{\prime}\left(y_{r}\right) q_{r}=y_{r} \chi_{\Theta} \text { in } Q_{r} \\
q_{r}=0 \text { on } \Sigma_{r} \\
q_{r}(., T)=0 \text { in } \Omega_{r} \\
\left\|q_{r}(0)\right\|_{L^{2}\left(\Omega_{r}\right)} \leq \varepsilon .
\end{array}\right.
$$

Observe that in fact this result provides, for each $r>0$ fixed, an $\varepsilon$-insensitizing control for the semilinear problem (1.4)(1.5) (in the restricted domain $\Omega_{r}$ ). We are going to prove that precisely these controls $\psi_{r}$ are uniformly bounded in $L^{2}(\omega \times(0, T))$.

Observe that Proposition 2.1 allows to prove this bound by just proving the following.

Proposition 4.1. Let $\bar{\zeta}_{r}$, $\bar{\eta}_{r}$ be the solutions of (4.1)-(4.2) corresponding to $F\left(y_{r}\right), f^{\prime}\left(y_{r}\right)$ with $y_{r}$ the fixed point of the mapping $\Lambda_{r}$ and data $y^{0}, \xi$ satisfying (H5). Then $\widetilde{\bar{\eta}}_{r}(0)$ is relatively compact in $L^{2}(\Omega)$ where $\widetilde{\bar{\eta}}_{r}(0)$ is the extension by zero of $\bar{\eta}_{r}(0)$ to $\Omega$.

Proof. First of all, since $f$ is globally Lipschitz we can proceed as in the proof of Lemma 3.3. We observe that $\gamma \geq \bar{\zeta}_{r}, \beta \geq \bar{\eta}_{r}$ for every $r>0$ where $\gamma, \beta$ satisfy

$$
\begin{gathered}
\left\{\begin{array}{l}
\gamma_{t}-\Delta \gamma-L \gamma=|\xi| \text { in } Q \\
\gamma=0 \text { on } \Sigma \\
\gamma(., 0)=\left|y^{0}\right| \text { in } \Omega
\end{array}\right. \\
\left\{\begin{array}{l}
-\beta_{t}-\Delta \beta-L \beta=\gamma \chi_{\Theta} \text { in } Q \\
\beta=0 \text { on } \Sigma \\
\beta(., T)=0 \text { in } \Omega .
\end{array}\right.
\end{gathered}
$$

with $L=\left|f^{\prime}\right|_{L^{\infty}}$.

On the other hand, it can also be proved that $\tilde{\gamma} \leq \bar{\zeta}_{r}, \tilde{\beta} \leq \bar{\eta}_{r}$ for every $r>0$ where $\tilde{\gamma}, \tilde{\beta}$ satisfy

$$
\begin{aligned}
& \left\{\begin{array}{l}
\tilde{\gamma}_{t}-\Delta \tilde{\gamma}-L \tilde{\gamma}=-|\xi| \text { in } Q \\
\tilde{\gamma}=0 \text { on } \Sigma \\
\tilde{\gamma}(., 0)=-\left|y^{0}\right| \text { in } \Omega
\end{array}\right. \\
& \left\{\begin{array}{l}
-\tilde{\beta}_{t}-\Delta \tilde{\beta}-L \tilde{\beta}=\tilde{\gamma} \chi_{\Theta} \text { in } Q \\
\tilde{\beta}=0 \text { on } \Sigma \\
\tilde{\beta}(., T)=0 \text { in } \Omega .
\end{array}\right.
\end{aligned}
$$


We observe that $\tilde{\beta} \leq 0 \leq \beta$ and then, if we put $\hat{\beta}=\beta-\tilde{\beta}$, we get that $\hat{\beta} \in L^{2}(\Omega \times(0, T))$ is such that for every $r>0$ and for every $t \in(0, T)$,

$$
\left|\bar{\eta}_{r}(x, t)\right| \leq \hat{\beta}(x, t) \text { a.e. in } \Omega_{r} \text {. }
$$

Since $\widetilde{\bar{\eta}}_{r}(0)$ is bounded in $L^{2}(\Omega)$ there exist $\eta \in L^{2}(\Omega)$ and a subsequence (still denoted by $r$ ) such that

$$
\widetilde{\bar{\eta}}_{r}(0) \rightarrow \eta \text { weakly in } L^{2}(\Omega) \text { as } r \rightarrow \infty .
$$

Proceeding as in the proof of Proposition 2.3 (see Section 6.2, Second step) we can see that for every compact $K \subset \Omega$ there exist $C>0$ independent of $r$ and $R$ large enough such that if $r>R$, then

$$
\left\|\nabla \bar{\eta}_{r}(0)\right\|_{L^{2}(K)} \leq C T^{-1 / 2} \text {. }
$$

Therefore we can find a subsequence with $r=r(K)$ and $\bar{\eta}_{K}$ such that

$$
\tilde{\bar{\eta}}_{r}(0) \rightarrow \bar{\eta}_{K} \text { strongly in } L^{2}(K) .
$$

But the uniqueness of the weak limit implies that $\bar{\eta}_{K}=\left.\eta\right|_{K}$. Since the limit has been identified in a unique way we have that

$$
\tilde{\bar{\eta}}_{r}(0) \rightarrow \eta \text { strongly in } L_{l o c}^{2}(\Omega) \text {. }
$$

On the other hand, in view of (4.8), for every $\delta>0$ there exists $R>0$ such that for every $r>R$ we have

$$
\left\|\widetilde{\bar{\eta}}_{r}(0)\right\|_{L^{2}\left(\Omega_{R}^{\prime}\right)} \leq\|\beta(0)\|_{L^{2}\left(\Omega_{R}^{\prime}\right)} \leq \delta / 3
$$

where $\Omega_{R}^{\prime}$ denotes $\Omega \backslash \Omega_{R}$, and for $R$ large enough $\|\eta\|_{L^{2}\left(\Omega_{R}^{\prime}\right)} \leq \delta / 3$. As a consequence of these facts we have for every $r>R$

$$
\left\|\widetilde{\bar{\eta}}_{r}(0)-\eta\right\|_{L^{2}(\Omega)} \leq\left\|\widetilde{\bar{\eta}}_{r}(0)-\eta\right\|_{L^{2}\left(\Omega_{R}\right)}+\frac{2 \delta}{3} .
$$

It is easy then to conclude that $\widetilde{\bar{\eta}}_{r}(0) \rightarrow \eta$ strongly in $L^{2}(\Omega)$.

\section{Proof of Theorem 1.5}

In the proof of Theorem 1.5 we need some previous results:

Proposition 5.1. Let $\varepsilon>0$ be fixed and assume that $1 \leq N \leq 6$. Assume that (H1)-(H4) hold and that $\xi \in L^{2}(Q), y^{0} \in L^{2}(\Omega)$. Let $\left\{\xi_{n}\right\} \subset L^{2}(Q)$, $\left\{y_{n}^{0}\right\} \subset L^{2}(\Omega)$ be sequences of functions with compact support such that

$$
\begin{array}{ccc}
\xi_{n} \rightarrow \xi & \text { strongly in } & L^{2}(Q) \\
y_{n}^{0} \rightarrow y^{0} & \text { strongly in } & L^{2}(\Omega) .
\end{array}
$$

Suppose that for every $n,\left\|h_{n}\right\|_{L^{2}(Q)} \leq H$, where $h_{n}$ are controls such that

$$
\left\|q_{n}(., 0)\right\|_{L^{2}(\Omega)} \leq \varepsilon / 2 \quad \forall n
$$

where $q_{n}$ is the corresponding solution of (1.5) with data $\xi_{n}, y_{n}^{0}$ and control $h_{n}$. Then there exists $\hat{n}>0$ such that the solution $q$ of (1.5) with $h=h_{\hat{n}}$ satisfies

$$
\|q(., 0)\|_{L^{2}(\Omega)} \leq \varepsilon .
$$

Furthermore, the same holds in space dimension $N \geq 7$ if $y^{0} \in L^{2}(\Omega) \cap$ $L^{N / 2}(\Omega), \xi \in L^{2}\left(0, T ; L^{2}(\Omega) \cap L^{N / 2}(\Omega)\right)$ and the sequences $\left\{y_{n}^{0}\right\} \subset L^{2}(\Omega) \cap$ Esaim: Cocv, June 1997, Vol. 2, Pr. 125-149 
$L^{N / 2}(\Omega),\left\{\xi_{n}\right\} \subset L^{2}\left(0, T ; L^{2}(\Omega) \cap L^{N / 2}(\Omega)\right)$ converge strongly to $y^{0}$, respectively to $\xi$, in these spaces.

Proof. Let $q$ be the solution of (1.11) with $h=h_{n}$ and $n$ to be chosen later. Then

$$
\|q(., 0)\|_{L^{2}(\Omega)} \leq\left\|q_{n}(., 0)\right\|_{L^{2}(\Omega)}+\left\|q(., 0)-q_{n}(., 0)\right\|_{L^{2}(\Omega)} .
$$

We are going to see that $n$ can be chosen such that

$$
\left\|q(., 0)-q_{n}(., 0)\right\|_{L^{2}(\Omega)} \leq \varepsilon / 2 .
$$

Let $y$ be the solution of (1.10) with $h=h_{n}$. Then $\psi_{n}=y-y_{n}$ and $\beta_{n}=q-q_{n}$ satisfy:

$$
\begin{aligned}
& \left\{\begin{array}{l}
\psi_{n, t}-\Delta \psi_{n}+f(y)-f\left(y_{n}\right)=\xi-\xi_{n} \text { in } Q \\
\psi_{n}=0 \text { on } \Sigma \\
\psi_{n}(., 0)=y^{0}-y_{n}^{0} \text { in } \Omega .
\end{array}\right. \\
& \left\{\begin{array}{l}
-\beta_{n, t}-\Delta \beta_{n}+f^{\prime}(y) q-f^{\prime}\left(y_{n}\right) q_{n}=\psi_{n} \chi_{\Theta} \text { in } Q \\
\beta_{n}=0 \text { on } \Sigma \\
\beta_{n}(., T)=0 \text { in } \Omega
\end{array}\right.
\end{aligned}
$$

We multiply (5.6) by $\beta_{n}$ and integrate by parts. Since $f^{\prime}(y) q-f^{\prime}\left(y_{n}\right) q_{n}=$ $q\left(f^{\prime}(y)-f^{\prime}\left(y_{n}\right)\right)+f^{\prime}\left(y_{n}\right)\left(q-q_{n}\right)$, we obtain:

$$
-\frac{1}{2} \frac{d}{d t} \int_{\Omega} \beta_{n}^{2}+\int_{\Omega}\left|\nabla \beta_{n}\right|^{2}-L \int_{\Omega}\left|\beta_{n}\right|^{2} \leq \int_{\Omega}\left|\psi_{n}\right|\left|\beta_{n}\right| \chi_{\Theta}+M\left|<q \psi_{n}, \beta_{n}>\right|
$$

where $<., .>$ denotes the duality product between $H^{-1}(\Omega)$ and $H_{0}^{1}(\Omega)$, $L=\left|f^{\prime}\right|_{L^{\infty}}$ and $M \geq\left|f^{\prime \prime}\right|_{L^{\infty}}$. Therefore

$$
-\frac{1}{2} \frac{d}{d t} \int_{\Omega}\left|\beta_{n}\right|^{2}+\frac{1}{2} \int_{\Omega}\left|\nabla \beta_{n}\right|^{2} \leq \frac{1}{2} \int_{\Omega}\left|\psi_{n}\right|^{2}+\frac{L}{2} \int_{\Omega} \beta_{n}^{2}+\frac{M^{2}}{2}\left\|q \psi_{n}\right\|_{H^{-1}(\Omega)}^{2} .
$$

By Gronwall's inequality we obtain

$$
\int_{\Omega}\left|\beta_{n}(0)\right|^{2} \leq C\left[\left\|\psi_{n}\right\|_{L^{2}(Q)}^{2}+\int_{0}^{T}\left\|q \psi_{n}\right\|_{H^{-1}(\Omega)}^{2}\right]
$$

In order to estimate $\left\|q \psi_{n}\right\|_{H^{-1}(\Omega)}^{2}$ we consider first the case $1 \leq N \leq 6$. We remember that $\psi_{n} \in L^{\infty}\left((0, T) ; L^{2}(\Omega)\right)$. Since $\Omega$ satisfies $(\mathrm{H} 2), q \in$ $L^{2}\left((0, T) ; H^{2}(\Omega)\right)$ (see $\left.[13]\right)$. Moreover, by the variation of constant formula it is not difficult to see that

$$
\left.\|q\|_{L^{2}\left(0, T ; H^{2}(\Omega)\right)}^{2} \leq C|\xi|_{L^{2}(Q)}^{2}+\left|y^{0}\right|_{L^{2}(\Omega)}^{2}+\left|h_{n}\right|_{L^{2}(\omega \times(0, T))}^{2}\right) .
$$

In view of Sobolev's embeddings, for $1 \leq N<4$, we have that $q \psi_{n} \in$ $L^{2}(Q) \subset L^{2}\left(0, T ; H^{-1}(\Omega)\right)$ with

$$
\int_{0}^{T}\left\|q \psi_{n}\right\|_{H^{-1}}^{2} \leq C\left\|\psi_{n}\right\|_{L^{\infty}\left(0, T ; L^{2}(\Omega)\right)}^{2}\|q\|_{L^{2}\left(0, T ; H^{2}(\Omega)\right)}^{2}
$$

and for $4 \leq N \leq 6, q \psi_{n} \in L^{2}\left(0, T ; H^{-1}(\Omega)\right)$ with

$$
\begin{gathered}
<q \psi_{n}, f>=\int_{\Omega} q \psi_{n} f d x \quad \forall f \in H_{0}^{1}(\Omega), \\
\int_{0}^{T}\left\|q \psi_{n}\right\|_{H^{-1}}^{2} \leq C\left\|\psi_{n}\right\|_{L^{\infty}\left(0, T ; L^{2}(\Omega)\right)}^{2}\|q\|_{L^{2}\left(0, T ; H^{2}(\Omega)\right)}^{2}
\end{gathered}
$$


For $N \geq 7$ we observe that $\psi_{n} \in L^{\infty}\left(0, T ; L^{2}(\Omega) \cap L^{N / 2}(\Omega)\right.$ ) (see [4] p. 46) and in view of Sobolev's embeddings $q \in L^{2}\left(0, T ; L^{\frac{2 N}{N-4}}(\Omega)\right)$, that is, $q \psi_{n} \in L^{2}(Q) \subset L^{2}\left(0, T ; H^{-1}(\Omega)\right)$ and

$$
\int_{0}^{T}\left\|q \psi_{n}\right\|_{H^{-1}}^{2} \leq C\left\|\psi_{n}\right\|_{L^{\infty}\left(0, T ; L^{N / 2}(\Omega)\right)}^{2}\|q\|_{L^{2}\left(0, T ; H^{2}(\Omega)\right)}^{2} .
$$

In view of (5.7) and (5.8) we obtain, for $1 \leq N \leq 6$, that

$$
\begin{aligned}
& \int_{\Omega}\left|\beta_{n}(0)\right|^{2} \\
& \leq C\left[\left\|\psi_{n}\right\|_{L^{\infty}\left(0, T ; L^{2}(\Omega)\right)}^{2}\left(1+\|\xi\|_{L^{2}(Q)}^{2}+\left\|y^{0}\right\|_{L^{2}(\Omega)}^{2}+\left\|h_{n}\right\|_{L^{2}(\omega \times(0, T))}^{2}\right)\right]
\end{aligned}
$$

and for $N \geq 7$

$$
\int_{\Omega}\left|\beta_{n}(0)\right|^{2} \leq C\left[\left\|\psi_{n}\right\|_{\infty, N 2}^{2}\left(1+\|\xi\|_{L^{2}(Q)}^{2}+\left\|y^{0}\right\|_{L^{2}(\Omega)}^{2}+\left\|h_{n}\right\|_{L^{2}(\omega \times(0, T))}^{2}\right)\right]
$$

where \|\|$_{\infty, N 2}$ denotes the norm in $L^{\infty}\left(0, T ; L^{2}(\Omega) \cap L^{N / 2}(\Omega)\right)$ We are assuming that the controls $h_{n}$ are uniformly bounded in $n$. Since $\xi, y^{0}$ are fixed we obtain for $1 \leq N \leq 6$

$$
\int_{\Omega}\left|\beta_{n}(0)\right|^{2} \leq D\left[\left\|\xi-\xi_{n}\right\|_{L^{2}(Q)}^{2}+\left\|y^{0}-y_{n}^{0}\right\|_{L^{2}(\Omega)}^{2}\right] .
$$

and for $N \geq 7$

$$
\int_{\Omega}\left|\beta_{n}(0)\right|^{2} \leq D\left[\left\|\xi-\xi_{n}\right\|_{L^{2}\left(0, T ; L^{2}(\Omega) \cap L^{N / 2}(\Omega)\right)}^{2}+\left\|y^{0}-y_{n}^{0}\right\|_{L^{2}(\Omega) \cap L^{N / 2}(\Omega)}^{2}\right] .
$$

In view of the convergence in (5.1) and in (5.2) it is clear that we can choose $\hat{n}$ such that

$$
\left\|q(., 0)-q_{\hat{n}}(., 0)\right\|_{L^{2}(\Omega)} \leq \varepsilon / 2 .
$$

We conclude the proof of Theorem 1.5 by giving a uniform bound for the controls $h_{n}$. The next proposition is a result in that direction.

Proposition 5.2. Let $\xi_{n}, y_{n}^{0}$ be as in Proposition 5.1 and $h_{n}$ be the control proposed in the previous section such that the solution $q_{n}$ of (1.11) satisfies

$$
\left\|q_{n}(., 0)\right\|_{L^{2}(\Omega)} \leq \varepsilon / 2 .
$$

Then there exists a constant $H>0$ independent of $n$, such that for every $n$

$$
\left\|h_{n}\right\|_{L^{2}(Q)} \leq H
$$

The proof of this proposition needs an analysis similar to that which we have done in the previous section. Observe that for each $n$, the control $h_{n}$ in the whole domain $\Omega$ is equal to a control in some restricted domain $\Omega_{r}$ for some $r$ large enough that depends on the data $y_{n}^{0}, \xi_{n}$. That is, $h_{n}=h_{r(n)}$. As in the previous section it is not difficult to see, having in mind Proposition 2.1, that the proof of Proposition 5.2 can be reduced to the following:

Proposition 5.3. Let $\bar{\zeta}_{r}^{n}, \bar{\eta}_{r}^{n}$ be the solutions of (4.1) (4.2) corresponding to $F\left(y_{r}^{n}\right), f^{\prime}\left(y_{r}^{n}\right)$ with $y_{r}^{n}$ the fixed point of the mapping $\Lambda_{r}$ and data $y_{n}^{0}, \xi^{n}$ satisfying the hypotheses of Proposition 5.1. Then $\widehat{\bar{\eta}_{r}^{n}(0)}$ is relatively compact in $L^{2}(\Omega)$ where $\widetilde{\bar{\eta}_{r}^{n}(0)}$ is the extension by zero of $\bar{\eta}_{r}^{n}(0)$ to $\Omega$.

Esaim: Cocv, June 1997, Vol. 2, Pr. 125-149 
Proof. Since the sequence $\xi_{n}$ has been chosen such that it converges strongly to $\xi$ in $L^{2}(Q)$ there exists $p \in L^{2}(Q)$ and a subsequence, still denoted by $n$, such that

$$
\left|\xi_{n}(x, t)\right| \leq p(x, t) \text { for almost every }(x, t) \in Q
$$

Since $y_{n}^{0}$ converges strongly to $y^{0}$ in $L^{2}(\Omega)$ there exist $Y \in L^{2}(\Omega)$ and a subsequence (of the previous one) such that

$$
\left|y_{n}^{0}(x)\right| \leq Y(x) \text { for almost every } x \in \Omega .
$$

Therefore, by the maximum principle, we have that for every $n$

$$
\bar{\zeta}_{r}^{n} \leq \gamma_{r} \quad, \quad \bar{\eta}_{r}^{n} \leq \beta_{r}
$$

where $\gamma_{r}$ and $\beta_{r}$ are solutions of

$$
\begin{aligned}
& \left\{\begin{array}{l}
\gamma_{r, t}-\Delta \gamma_{r}+F\left(y_{r}^{n}\right) \gamma_{r}=p(x, t) \chi_{Q_{r}} \text { in } Q_{r} \\
\gamma_{r}=0 \text { on } \Sigma_{r} \\
\gamma_{r}(., 0)=Y(x) \chi_{\Omega_{r}} \text { in } \Omega_{r}
\end{array}\right. \\
& \left\{\begin{array}{l}
-\beta_{r, t}-\Delta \beta_{r}+f^{\prime}\left(y_{r}^{n}\right) \beta_{r}=\gamma_{r} \chi_{\Theta} \\
\beta_{r}=0 \text { on } \Sigma_{r} \\
\beta_{r}(., T)=0 \text { in } Q_{r} .
\end{array}\right.
\end{aligned}
$$

We proceed as in the proof of Proposition 4.1. That is, we can find $\beta \in L^{2}(\Omega \times(0, T))$ such that for every $n>0$ and for every $r>0$

$$
\left|\bar{\eta}_{r}^{n}(x, t)\right| \leq\left|\beta_{r}(x, t)\right| \leq \beta(x, t) \text { in } \Omega_{r} \times(0, T) .
$$

Therefore $\left\|\widetilde{\bar{\eta}_{r}^{n}}(0)\right\|_{L^{2}(\Omega)} \leq C$ and we can find $\eta \in L^{2}(\Omega)$ and a subsequence $r(n)$, with $r(n) \rightarrow \infty$ as $n \rightarrow \infty$, such that

$$
\widetilde{\overline{\eta_{r(n)}}}(0) \rightarrow \eta \text { weakly in } L^{2}(\Omega) \text { as } r(n) \rightarrow \infty \text {. }
$$

Proceeding as in the proof of Proposition 4.1 we conclude that

$\widetilde{\bar{\eta}_{r(n)}}(0) \rightarrow \eta$ strongly in $L^{2}(\Omega)$.

\section{TEChNical PROOFS}

\subsection{Proof of Lemma 3.3}

Let $X$ be solution of (3.14). Since $X(0)=\left|y^{0}\right| \geq 0$ and $|g| \geq 0$, by the maximum principle $X$ is positive. Moreover $|F(Y)|<M Y$ with $M$ the Lipschitz constant of $F$, and then $X$ is subsolution of the problem

$$
\left\{\begin{array}{l}
u_{t}-\Delta u-M u=|g| \text { in } \mathbb{R}^{n} \times(0, T) \\
u(x, 0)=\left|y^{0}(x)\right| \text { in } \mathbb{R}^{n}
\end{array}\right.
$$

Let $v=e^{-M t} u$. Then $v$ verifies

$$
\left\{\begin{array}{l}
v_{t}-\Delta v=e^{-M t}|g| \text { in } \mathbb{R}^{n} \times(0, T) \\
v(x, 0)=\left|y^{0}(x)\right| \text { in } \mathbb{R}^{n}
\end{array}\right.
$$

We can express $v$ by the variation of constants formula:

$$
\begin{aligned}
v(x, t) & =\int_{\mathbb{R}^{n}}(4 \pi t)^{-n / 2} e^{\frac{-|x-z|^{2}}{4 t}}\left|y^{0}(z)\right| d z \\
& +\int_{0}^{t} \int_{|z|<\rho}(4 \pi(t-\sigma))^{-n / 2} e^{\frac{-|x-z|^{2}}{4(t-\sigma)}} e^{-M \sigma}|g(\sigma, z)| d \sigma d z
\end{aligned}
$$


Since supp $y^{0} \subset B_{\rho}, \omega \subset B_{\rho}$ and supp $g \subset B_{\rho} \times(0, T)$ we have, for every $x$ such that $|x|>\rho$,

$$
\begin{aligned}
v(x, t) & \leq \int_{|z|<\rho}(4 \pi t)^{-n / 2} e^{\frac{-\| x|-\rho|^{2}}{4 t}}\left|y^{0}(z)\right| d z \\
& +\int_{0}^{t} \int_{|z|<\rho}(4 \pi(t-\sigma))^{-n / 2} e^{\frac{-|| x|-\rho|^{2}}{4(t-\sigma)}}|g(\sigma, z)| d \sigma d z .
\end{aligned}
$$

and then

$$
\begin{aligned}
v(x, t) & \leq(4 \pi t)^{-n / 2} e^{\frac{-|| x|-\rho|^{2}}{4 t}}\left|y^{0}\right|_{L^{1}\left(B_{\rho}\right)} \\
& +\int_{0}^{t} \int_{|z|<\rho}(4 \pi(t-\sigma))^{-n / 2} e^{\frac{-\| x|-\rho|^{2}}{4(t-\sigma)}}|g(\sigma, z)| d \sigma d z
\end{aligned}
$$

Let $m(s)=s^{-\alpha} e^{-\beta / s}$. By elementary calculus we obtain that $m$ reaches it maximum at $s=\frac{\beta}{\alpha}$. Then

$$
s^{-\alpha} e^{-\beta / s} \leq\left(\frac{e}{\alpha}\right)^{-\alpha} \beta^{-\alpha} .
$$

Substituting this bound in (6.3) with $\alpha=n / 2$ and $\beta=|| x|-\rho|^{2}$, we obtain

$$
\|v\|_{L^{\infty}\left(\Gamma_{r}\right)} \leq\left(\frac{2 \pi e}{n}\right)^{-n / 2} \frac{1}{(r-\rho)^{n}}\left(\left\|y^{0}\right\|_{L^{1}\left(\mathbb{R}^{n}\right)}+\|g\|_{L^{1}\left((0, T) \times \mathbb{R}^{n}\right)}\right) .
$$

Since $u(x, t)=e^{M t} v \leq e^{M T} v$ we have that

$$
\|u\|_{L^{\infty}\left(\Gamma_{r}\right)} \leq C \frac{e^{M T}}{(r-\rho)^{n}}\left(\left\|y^{0}\right\|_{L^{1}\left(\mathbb{R}^{n}\right)}+\|g\|_{L^{1}\left((0, T) \times \mathbb{R}^{n}\right)}\right) . \quad \forall r>\rho .
$$

Since $X$ is a non negative subsolution of (6.1)

$$
\|X\|_{L^{\infty}\left(\Gamma_{r}\right)} \leq C \frac{e^{M T}}{(r-\rho)^{n}}\left(\left\|y^{0}\right\|_{L^{1}\left(\mathbb{R}^{n}\right)}+\|g\|_{L^{1}\left((0, T) \times \mathbb{R}^{n}\right)}\right) . \forall r>\rho .
$$

\subsection{Proof of Proposition 2.3}

This section is devoted to the proof of Proposition 2.3. During the proof we are going to use the following classical compactness result (see Simon [12], Theorem 5, p. 84):

Theorem 6.1. Let $X, B, Y$ be Banach spaces such that $X \subset B \subset Y$ with continuous embeddings, the embedding $X \subset B$ being compact. Let $1 \leq p \leq$ $\infty$. If $\mathcal{F}$ is a bounded subset of $L^{p}(0, T ; X)$ and

$$
\left\|\tau_{h} f-f\right\|_{L^{p}(0, T-h ; Y)} \rightarrow 0 \text { as } h \rightarrow 0 \text { uniformly for } f \in \mathcal{F}
$$

where $\tau_{h} f(t)=f(t+h)$, then $\mathcal{F}$ is relatively compact in $L^{p}(0, T ; B)$ [in $C([0, T] ; B)$ if $p=\infty$.

Proof of Proposition 2.3. First of all we observe that since $\gamma_{r}^{0} \in L^{2}\left(\Omega_{r}\right)$, $g_{r} \in L^{2}\left(Q_{r}\right)$ and $\gamma_{r} \in L^{2}\left(0, T ; H_{0}^{1}\left(\Omega_{r}\right)\right)$, then $\nabla \widetilde{\gamma_{r}}=\widetilde{\nabla \gamma_{r}}$ and $\widetilde{\gamma_{r}}$ satisfies

$$
\left\{\begin{array}{l}
\widetilde{\gamma_{r}, t}-\Delta \widetilde{\gamma_{r}}+\widetilde{c_{r}}(x, t) \widetilde{\gamma_{r}}=\widetilde{g_{r}}+\frac{\partial \gamma_{r}}{\partial \nu} \delta_{\partial \Omega_{r}} \text { in } \mathrm{Q} \\
\widetilde{\gamma_{r}}=0 \text { on } \Sigma \\
\widetilde{\gamma_{r}}(., 0)=\widetilde{\gamma_{r}^{0}} \text { in } \Omega
\end{array}\right.
$$


where $\delta_{\partial \Omega_{r}}$ is the Dirac measure on the set $\partial \Omega_{r}$. That is, for $\phi \in C^{0}(\bar{\Omega})$, $<\frac{\partial \gamma_{r}(t)}{\partial \nu} \delta_{\partial \Omega_{r}}, \phi>=\int_{\partial \Omega_{r}} \frac{\partial \gamma_{r}(t)}{\partial \nu} \phi d \sigma$. In view of (6.4) we have that for every compact subset $K \subset \Omega, R>0$ such that $K \subset B_{R}$ and for every $r>R$,

$$
\widetilde{\gamma_{r}, t}=\Delta \widetilde{\gamma_{r}}-\widetilde{c_{r}}(x, t) \widetilde{\gamma_{r}}+\widetilde{g_{r}} \text { in } K \times(0, T) \text {. }
$$

On the other hand we have that

$$
\begin{gathered}
\left\|\widetilde{\gamma_{r}^{0}}\right\|_{L^{2}(\Omega)}=\left\|\gamma_{r}^{0}\right\|_{L^{2}\left(\Omega_{r}\right)} \leq C \forall r \\
\left\|g_{r}\right\|_{L^{2}\left(Q_{r}\right)}=\left\|\widetilde{g_{r}}\right\|_{L^{2}(Q)} \leq T^{1 / 2}\left\|\widetilde{g_{r}}\right\|_{L^{\infty}\left(0, T ; L^{2}(\Omega)\right)} \leq C \forall r .
\end{gathered}
$$

In consequence there exist $\gamma^{0} \in L^{2}(\Omega), g \in L^{2}(Q)$ and subsequences (with the same index $r$ ) such that

$$
\begin{gathered}
\widetilde{\gamma_{r}^{0}} \rightarrow \gamma^{0} \text { weakly in } L^{2}(\Omega) \text { as } r \rightarrow \infty, \\
\widetilde{g_{r}} \rightarrow g \text { weakly in } L^{2}(Q) \text { as } r \rightarrow \infty .
\end{gathered}
$$

We shall see that the result stated in Proposition 2.3 holds precisely for the sequences corresponding to that index $r$.

We divide now the proof in 5 steps. The first three correspond to convergence results, the fourth step is devoted to prove that $\gamma$ verifies equation (2.13) and the fifth that $\gamma$ belongs to $L_{l o c}^{2}\left(0, T ; H_{l o c}^{2}(\Omega)\right)$.

FiRST STEP: $\widetilde{\gamma_{r}} \rightarrow \gamma$ WEAKLY ${ }^{*}$ IN $L^{\infty}\left(0, T ; L^{2}(\Omega)\right)$.

We multiply equation $(2.6)$ by $\gamma_{r}$ and integrate by parts

$$
\frac{1}{2} \frac{d}{d t} \int_{\Omega_{r}}\left|\gamma_{r}\right|^{2} d x+\int_{\Omega_{r}}\left|\nabla \gamma_{r}\right|^{2}+\int_{\Omega_{r}} c_{r}(x, t)\left|\gamma_{r}\right|^{2}=\int_{\Omega_{r}} g_{r} \gamma_{r} .
$$

Since $\left|c_{r}\right|$ is uniformly bounded in $L^{\infty}(Q), \widetilde{g}_{r}$ is uniformly bounded in $L^{2}(Q)$ and $\widetilde{\gamma_{r}^{0}}$ is uniformly bounded in $L^{2}(\Omega)$ we can apply first Schwartz's inequality and then Gronwall's inequality to obtain that

$$
\int_{\Omega_{r}}\left|\gamma_{r}(t)\right|^{2} d x+2 \int_{0}^{t} \int_{\Omega_{r}}\left|\nabla \gamma_{r}\right|^{2} d x d s \leq C
$$

for some constant $C>0$ independent of $t$ and $r$. Therefore, for every $r$

$$
\begin{gathered}
\int_{\Omega_{r}}\left|\gamma_{r}(t)\right|^{2} d x \leq C \forall t \in(0, T) \\
\int_{0}^{t} \int_{\Omega_{r}}\left|\nabla \gamma_{r}\right|^{2} \leq C \forall t \in(0, T) .
\end{gathered}
$$

In view of (6.10) we can extract a subsequence (from that we have chosen in $(6.8))$ verifying

$$
\widetilde{\gamma_{r}} \rightarrow \gamma \text { weakly* in } L^{\infty}\left(0, T ; L^{2}(\Omega)\right),
$$

and in particular

$$
\widetilde{\gamma_{r}} \rightarrow \gamma \text { weakly in } L^{3}\left(0, T ; L^{2}(\Omega)\right) .
$$

SECOND Step: $\widetilde{\gamma_{r}(t)} \rightarrow \gamma(t)$ STRONGLy in $L_{l o c}^{2}(\Omega)$ FOR EVERy $t$.

For $K \subset \Omega$ compact, from (6.5) and (6.10) we have

$$
\left\|\widetilde{\gamma_{r}, t}\right\|_{L^{\infty}\left(0, T ; H^{-2}(K)\right)} \leq C \text {. }
$$


In view of Theorem 6.1, taking $B=H^{-1}(K), X=L^{2}(K), Y=H^{-2}(K)$, $p=\infty$ and $F=\left\{\widetilde{\gamma}_{r}\right\}_{r}$, there exists $\rho(K)$ and a subsequence $\tilde{\gamma}_{r}$ (from the previous one) such that $\widetilde{\gamma_{r}} \rightarrow \rho(K)$ strongly in $C\left([0, T] ; H^{-1}(K)\right)$.

By uniqueness of the weak limit and extracting diagonal subsequences we observe that for every compact subset $K, \rho(K)=\left.\gamma\right|_{K}$, i.e.

$$
\widetilde{\gamma_{r}} \rightarrow \gamma \text { strongly in } C\left([0, T] ; H_{l o c}^{-1}(\Omega)\right)
$$

and $\left.\gamma^{0}\right|_{K}=\left.\gamma(0)\right|_{K}$

In order to get compactness in $L^{2}$ we need some estimates on the gradients. Let $S_{r}(t)$ be the semigroup generated by the operator $-\Delta$ in $L^{2}\left(\Omega_{r}\right)$ with Dirichlet boundary conditions. Since $S_{r}(\cdot)$ is an analytic semigroup we have (see [10]) that

$$
\left\|S_{r}(t) v\right\|_{H^{1}\left(\Omega_{r}\right)} \leq t^{-1 / 2}\|v\|_{L^{2}\left(\Omega_{r}\right)}, \quad \forall v \in L^{2}\left(\Omega_{r}\right) .
$$

By the variation of constants formula, we know that

$$
\begin{aligned}
\gamma_{r}(t)= & S_{r}(t) \gamma_{r}(0)-\int_{0}^{t} S_{r}(t-\sigma) c_{r}(\sigma) \gamma_{r}(\sigma) d \sigma \\
& +\int_{0}^{t} S_{r}(t-\sigma) g_{r}(\sigma) d \sigma
\end{aligned}
$$

It is not difficult to prove then that

$$
\left\|\nabla \gamma_{r}(t)\right\|_{L^{2}(K)} \leq\left\|\nabla \gamma_{r}(t)\right\|_{L^{2}\left(\Omega_{r}\right)} \leq C t^{-1 / 2}\left\|\gamma_{r}^{0}\right\|_{L^{2}\left(\Omega_{r}\right)} \text { for every } \quad 0<t \leq T
$$

where $C$ depends only of $\left\|\widetilde{c}_{r}\right\|_{L^{\infty}\left(Q_{r}\right)}$ and $\left\|\widetilde{g}_{r}\right\|_{L^{\infty}\left(0, T ; L^{2}\left(\Omega_{r}\right)\right)}$. In view of our hypotheses on $c_{r}, g_{r}$ and $\gamma_{r}^{0}$ it is easy to see then that

$$
\left\|\nabla \gamma_{r}(t)\right\|_{L^{2}(K)} \leq C t^{-1 / 2} \text { for every } 0<t \leq T
$$

with $C$ independent of $r$.

In view of (6.16), for every $t \in(\varepsilon, T)$ there exists $r_{i}=r_{i}(t)\left[r_{i}\right.$ subsequence of $(6.14)]$ and $\eta(t ; K)$ such that $\widehat{\gamma_{r_{i}}}(t) \rightarrow \eta(t ; K)$ strongly in $L^{2}(K)$. In view of $(6.14) \eta(t ; K)=\left.\gamma(t)\right|_{K}$ for $t \in(\varepsilon, T)$ and therefore $\left.\widetilde{\gamma_{r_{i}}}(t) \rightarrow \gamma(t)\right|_{K}$ strongly in $L^{2}(K)$.

The subsequence $r_{i}$ depends (in principle) on $t$. However, since the limit has been identified in a unique way as $\left.\gamma(t)\right|_{K}$ we deduce that the whole sequence converges, i.e. for every compact $K \subset \Omega$ and $0<t<T, \widetilde{\gamma_{r}}(t) \rightarrow$ $\gamma(t)$ in $L^{2}(K)$.

THIRD STEP: $\widetilde{\gamma_{r}} \rightarrow \gamma$ IN $L^{2}\left(\varepsilon, T ; L_{l o c}^{2}(\Omega)\right)$.

Let $0 \leq \varepsilon<T$ and $\eta_{r}(t)=\left\|\tilde{\gamma_{r}}(t)-\gamma(t)\right\|_{L^{2}(K)}$. From (6.13) we know that there exists a constant $C>0$ such that

$$
\left(\int_{\varepsilon}^{T}\left|\eta_{r}(t)\right|^{3}\right)^{1 / 3} \leq C .
$$

Since $\left.\widetilde{\gamma_{r}}(t) \rightarrow \gamma(t)\right|_{K}$ strongly in $L^{2}(K)$ and by Egorov's Theorem, for any $\delta>0$ there exists a set $B_{\delta} \subset[\varepsilon, T]$ such that $A_{\delta}=[\varepsilon, T] \backslash B_{\delta}$ with measure $\left|A_{\delta}\right|<\delta^{3} / C^{6}$ and such that $\eta_{r} \rightarrow 0$ uniformly in $B_{\delta}$. Let $R$ be such that for Esaim: Cocv, June 1997, Vol. 2, Pr. 125-149 
every $r>R,\left|\eta_{r}\right|<\left|\frac{\delta}{\left|B_{\delta}\right|}\right|^{1 / 2}$ in $B_{\delta}$. Then, for every $r>R$ we have

$$
\int_{\varepsilon}^{T}\left|\eta_{r}(t)\right|^{2} \leq \int_{A_{\delta}}\left|\eta_{r}(t)\right|^{2}+\int_{B_{\delta}}\left|\eta_{r}(t)\right|^{2} \leq C^{2} \frac{\delta}{C^{2}}+\left|B_{\delta}\right| \frac{\delta}{\left|B_{\delta}\right|}=2 \delta .
$$

Therefore $\left.\widetilde{\gamma_{r}} \rightarrow \gamma\right|_{K}$ strongly in $L^{2}\left(\varepsilon, T ; L^{2}(K)\right)$. Clearly, the limit $\gamma$ does not depend on the compact set $K \subset \Omega$. Thus

$$
\widetilde{\gamma_{r}} \rightarrow \gamma \text { strongly in } L^{2}\left(\varepsilon, T ; L_{l o c}^{2}(\Omega)\right) \text {. }
$$

Fourth STEP: EQUATION VERIFIED BY $\gamma$.

Let $v \in \mathcal{D}(Q)$ and $R>0$ be such that supp $v \subset B_{R} \times(\varepsilon, T)$. Then for every $r>R$

$$
-\int_{0}^{T} \int_{\Omega} \tilde{\gamma_{r}} v_{t}-\int_{0}^{T} \int_{\Omega} \tilde{\gamma_{r}} \Delta v+\int_{0}^{T} \int_{\Omega} \widetilde{c_{r}} \widetilde{\gamma_{r}} v=\int_{0}^{T} \int_{\Omega} \widetilde{g_{r}} v
$$

Since $\left|\widetilde{c_{r}}\right|_{L^{\infty}(Q)} \leq C$, there exists a sequence (still denoted by $r$ ) and $c \in$ $L^{\infty}(Q)$ such that $\widetilde{c_{r}} \rightarrow c$ weakly* in $L^{\infty}(Q)$. On the other hand, $\widetilde{g_{r}} \rightarrow g$ weakly in $L^{2}(Q)$. Passing to the limit in (6.17) along the subsequence we obtain

$$
-\int_{0}^{T} \int_{\Omega} \gamma v_{t}-\int_{0}^{T} \int_{\Omega} \gamma \Delta v+\int_{0}^{T} \int_{\Omega} c(x, t) \gamma v=\int_{0}^{T} \int_{\Omega} g v
$$

and therefore $\gamma$ verifies the equation

$$
\gamma_{t}-\Delta \gamma+c(x, t) \gamma=g \text { in } \mathcal{D}^{\prime}(Q)
$$

where $\mathcal{D}^{\prime}(Q)$ denotes the set of all the distributions in $Q$.

FifTH STEP: $\gamma$ BELONGs To $L_{l o c}^{2}\left(0, T ; H_{l o c}^{2}(\Omega)\right)$.

In order to estimate the norm of the solution in $L^{2}\left(\varepsilon, T ; H^{2}(K)\right)$ we need to introduce some auxiliary sets and functions. Given any $\delta>0$ we choose $R$ and $K_{R} \subset \Omega_{R}$ an open set of class $C^{2}$ (we observe that $\Omega_{R}=\Omega \cap B_{R}$ has not necessarily this regularity) such that $\operatorname{dist}\left(\partial K, \partial K_{R}\right)>3 \delta>0$. Let $K_{\delta}$ be a compact set of class $C^{2}$ such that $K \subset K_{\delta} \subset K_{R}$ and $\operatorname{dist}\left(\partial K_{\delta}, \partial K_{R}\right)>\delta$. We construct $\phi \in C^{\infty}(\Omega)$ such that $\phi=1$ in $K_{\delta}$ and $\phi=0$ in $\Omega \backslash K_{R} \cup \partial K_{R}$. For every $r>R$ we define

$$
U_{r}=\gamma_{r} \phi
$$

Then $U_{r}$ satisfies

$$
\left\{\begin{array}{l}
U_{r, t}-\Delta U_{r}+c_{r}(x, t) U_{r}=\gamma_{r} \Delta \phi-2 \operatorname{div}\left(\gamma_{r} \nabla \phi\right)+\phi g_{r} \text { in } K_{R} \times(0, T) \\
U_{r}=0 \text { on } \partial K_{R} \times(0, T) \\
U_{r}()=\gamma_{r}^{0} \phi \text { in } K_{R} .
\end{array}\right.
$$

Let $S(t)$ be the semigroup generated by the operator $-\Delta$ in $L^{2}\left(K_{R}\right)$ with Dirichlet boundary conditions. By the variation of constants formula we observe that for every $0 \leq t<T$,

$$
\begin{aligned}
U_{r}(t)= & S(t) U_{r}(0)+\int_{0}^{t} S(t-\sigma) \gamma_{r}(\sigma) \Delta \phi d \sigma \\
& -2 \int_{0}^{t} S(t-\sigma) \operatorname{div}\left(\gamma_{r}(\sigma) \nabla \phi\right) d \sigma-\int_{0}^{t} S(t-\sigma) c_{r}(\sigma) U_{r}(\sigma) d \sigma \\
& +\int_{0}^{t} S(t-\sigma) g_{r}(\sigma) \phi(\sigma) d \sigma
\end{aligned}
$$


Since $S(\cdot)$ is an analytic semigroup and $K_{R}$ is of class $C^{2}$, we have (see [10], p.74)

$$
\|S(t) v\|_{L^{2}\left(K_{R}\right)} \leq\|v\|_{L^{2}\left(K_{R}\right)} ;\|S(t) v\|_{H^{2}\left(K_{R}\right)} \leq \frac{1}{t}\|v\|_{L^{2}\left(K_{R}\right)}, \forall v \in L^{2}\left(K_{R}\right)
$$

Interpolating for $0<s<2$ (see Lions-Magenes [9]) we obtain

$$
\|S(t) v\|_{H^{s}\left(K_{R}\right)} \leq C(s) t^{-s / 2}\|v\|_{L^{2}\left(K_{R}\right)}, \forall v \in L^{2}\left(K_{R}\right)
$$

Therefore,

$$
\begin{aligned}
\left\|U_{r}(t)\right\|_{H^{3 / 2}\left(K_{R}\right) \leq} \leq & C\left(K_{R}\right) t^{-3 / 4}\left\|U_{r}(0)\right\|_{L^{2}\left(K_{R}\right)} \\
& +C\left(K_{R}, \phi\right)\left\|\gamma_{r}\right\|_{L^{\infty}\left(0, T ; L^{2}\left(\Omega_{r}\right)\right)} \int_{0}^{t}(t-\sigma)^{-3 / 4} d \sigma \\
& +C\left(K_{R}, \phi\right)\left\|\gamma_{r}\right\|_{L^{\infty}\left(0, T ; L^{2}\left(\Omega_{r}\right)\right)} \int_{0}^{t}(t-\sigma)^{-3 / 4} \sigma^{-1 / 2} d \sigma \\
& +C\left(K_{R}, C\right)\left\|U_{r}\right\|_{L^{\infty}\left(0, T ; L^{2}\left(K_{R}\right)\right)} \int_{0}^{t}(t-\sigma)^{-3 / 4} d \sigma \\
& +C\left(K_{R}, \phi\right)\left\|g_{r}\right\|_{L^{\infty}\left(0, T ; L^{2}\left(K_{R}\right)\right)} \int_{0}^{t}(t-\sigma)^{-3 / 4} d \sigma .
\end{aligned}
$$

In view of $(6.6),(6.7),(6.10)$ and $(6.19)$ we obtain

$$
\left\|U_{r}(t)\right\|_{H^{3 / 2}\left(K_{R}\right)} \leq C\left[t^{-3 / 4}+1\right]
$$

where the constant $C$ depends on $\phi, K_{R}, T, G \geq\left\|g_{r}\right\|_{L^{\infty}\left(0, T ; L^{2}(\Omega)\right)}$ and $C \geq$ $\left\|c_{r}\right\|_{\infty}$. That is $\left\|\gamma_{r}(t)\right\|_{H^{3 / 2}\left(K_{\delta}\right)} \leq C\left[t^{-3 / 4}+1\right]$.

By a bootstrap argument we obtain that

$$
\int_{\varepsilon}^{T}\left\|\gamma_{r}(t)\right\|_{H^{2}(K)}^{2} \leq C
$$

and therefore (for a subsequence) $\left.\left.\widetilde{\gamma_{r}}\right|_{K} \rightarrow \gamma\right|_{K}$ weakly in $L^{2}\left(\varepsilon, T ; H^{2}(K)\right)$. This concludes the proof of Proposition 2.3.

\section{Appendix A. Proof of Proposition 1.1}

We give an sketch of the proof of Proposition 1.1.

The state $z$ solution of (1.1) is differentiable with respect to $\tau_{0}$ and it's derivative $z_{\tau_{0}}$ satisfies:

$$
\left\{\begin{array}{l}
z_{\tau_{0}, t}-\Delta z_{\tau_{0}}+f^{\prime}(y) z_{\tau_{0}}=0 \text { in } Q=\Omega \times(0, T) \\
z_{\tau_{0}}=0 \text { on } \Sigma=\partial \Omega \times(0, T) \\
z_{\tau_{0}}(x, 0)=z^{0} \text { in } \Omega
\end{array}\right.
$$

where $y$ is the solution of (1.4). The derivative of $\Phi$ with respect to $\tau_{0}$ at $\tau_{0}=0$ is then

$$
\left.\frac{\partial \Phi}{\partial \tau_{0}}\right|_{\tau_{0}=0}=\int_{0}^{T} \int_{\theta} y z_{\tau_{0}} d x d t .
$$

Substituting $y$ in (A.2) by left hand member of (1.5) and integrating by parts we obtain:

$$
\left.\frac{\partial \Phi}{\partial \tau_{0}}\right|_{\tau_{0}=0}=\int_{\Omega} q(x, 0) z^{0}(x) d x
$$


obtaining the condition (1.6).

\section{REFERENCES}

[1] S. Angenent: The zero set of a solution of a parabolic equation, J. Reine Angew. Math. ,390, 1988, 79-96.

[2] O. Bodart, C. Fabre: Controls Insensitizing the Norm of the Solution of a Semilinear Heat Equation, J. Math. An. and App., 195, 1995, 658-683.

[3] F.E. Browder: Estimates and existence theorems for elliptic boundary value problems. Proc. N. A. S., 45, 1959, 365-375.

[4] T. Cazenave, A. Haraux: Introduction aux problèmes d'évolution semi-linéaires, Collection S.M.A.I. Mathématiques et applications, Ellipses, Paris, 1980.

[5] L. de Teresa, E. Zuazua: Approximate controllability of a semilinear heat equation in unbounded domains, Preprint.

[6] C. Fabre, J.P. Puel, E. Zuazua: Approximate controllability of the semilinear heat equation, Proc. Roy. Soc. Edinburgh, Sect. A, 125, 1995, 31-61.

[7] O.A. Ladyzenskaja, V.A. Solonnikov, N.N. Ural'ceva: Linear and Quasilinear Equations of Parabolic Type, A.M.S., Rhode Island, 1968.

[8] J.L. Lions: Remarques préliminaires sur le contrôle des systèmes à données incomplètes, in Actas del Congreso de Ecuaciones Diferenciales y Aplicaciones (CEDYA), Universidad de Málaga, 1989, 43-54.

[9] J.L Lions, E. Magenes: Problèmes aux limites non homogènes et applications. Vol I \& II, Dunod, Paris, 1968

[10] A. Pazy : Semigroups of Linear Operators and Applications to Partial Differential Equations, Springer-Verlag, New York, 1983.

[11] J.C. Saut, B. Scheurer: Unique continuation for some evolution equations, J. Diff. Equations, 66(1), 1987, 118-139.

[12] J. Simon: Compact sets in the space $L^{p}(0, T ; B)$, Annali di Matematica Pura ed Applicata, CXLVI, (IV), 1987, 1173-1191.

[13] H. Tanabe: Equations of Evolution, Pitman, Bath, 1979.

[14] H. Triebel: Interpolation Theory, Function Spaces, Differential Operators, NorthHolland, Berlin, 1978. 